\title{
Signals of leptophilic dark matter at the ILC
}

\author{
Sukanta Dutta ${ }^{1, \mathrm{a}}$, Bharti Rawat ${ }^{2, \mathrm{~b}}$, Divya Sachdeva ${ }^{2, \mathrm{c}}$ \\ ${ }^{1}$ SGTB Khalsa College, University of Delhi, Delhi 110007, India \\ 2 Department of Physics and Astrophysics, University of Delhi, Delhi 110007, India
}

Received: 27 April 2017 / Accepted: 2 September 2017 / Published online: 22 September 2017

(C) The Author(s) 2017. This article is an open access publication

\begin{abstract}
Adopting a model independent approach, we constrain the various effective interactions of leptophilic DM particles with the visible world from the WMAP and Planck data. The thermally averaged indirect DM annihilation cross section and the DM-electron direct-detection cross section for such a DM candidate are observed to be consistent with the respective experimental data. We study the production of cosmologically allowed leptophilic DM in association with $Z(Z \rightarrow f \bar{f}), f \equiv q, e^{-}, \mu^{-}$at the ILC. We perform the $\chi^{2}$ analysis and compute the $99 \%$ C.L. acceptance contours in the $m_{\chi}$ and $\Lambda$ plane from the two-dimensional differential distributions of various kinematic observables obtained after employing parton showering and hadronisation to the simulated data. We observe that the dominant hadronic channel provides the best kinematic reach of $2.62 \mathrm{TeV}\left(m_{\chi}=25\right.$ $\mathrm{GeV}$ ), which further improves to $\sim 3 \mathrm{TeV}$ for polarised beams at $\sqrt{s}=1 \mathrm{TeV}$ and an integrated luminosity of $1 \mathrm{ab}^{-1}$.
\end{abstract}

\section{Introduction}

Dark matter provides the most compelling explanation for many cosmological and astrophysical observations which defy an understanding in terms of luminous matter alone. However, in the absence of any direct evidence, the existence of such matter has, rightly, been questioned and several alternatives have been proposed, including the modification of gravity at large distance scales [1]. It should be appreciated, though, that starting with orbital velocities of stars in a galaxy or those of the galaxies themselves in a cluster [2], gravitational lensing [3,4], the dynamics of galactic (cluster) collisions [5] etc., the observations span a wide range of distance scales, and no single simple modification of gravitation can explain all, whereas dark matter (DM) can and does play

\footnotetext{
a e-mail: Sukanta.Dutta@gmail.com

b e-mail: bhartirawat87@gmail.com

c e-mail: divyasachdeva951@gmail.com
}

an important role in understanding the data. Similarly, the fitting of the cosmological observables [6-8], requires that DM contributes about $23 \%$ to the energy budget of the universe, in contrast to only $4 \%$ contained in baryonic matter. Finally, post-inflation, perturbations in the DM distribution, along with the gravitational perturbations, are supposed to have provided the seed for large-scale structure formation in the universe [7]. The constraints from the latter are rather strong. Indeed, while neutrinos would have been natural candidates for DM within the Standard Model (SM), a large energy component in neutrinos would have disrupted structure formation. For example, a recent study claims that if the equation of state for the DM be parametrised as $p=w \rho$, then the combined fitting of the cosmic microwave background, the baryon acoustic oscillation data and the Hubble telescope data restricts $-9.0 \times 10^{-3}<w<2.4 \times 10^{-3}$, thereby clearly preferring a cold and dusty DM [9].

With the DM particle, by definition, not being allowed to have either electromagnetic ${ }^{1}$ or strong interaction, the most popular candidate is the weakly interacting massive particle (WIMP). And while its exact nature is unknown, several theoretical scenarios such as multi-Higgs models, supersymmetry, extra-dimensional theories, little Higgs models, leftright symmetric models all naturally admit viable candidates. Consequently, one of the most challenging tasks today is to identify the nature of the DM particle [11]. This, in principle, could be done in three kind of experiments. Direct detection can be achieved by setting up apparatuses (very often ultra-cold bolometric devices) that would register the scattering of a DM particle off the detector material. While the DAMA experiment [12] did indeed claim the existence of a DM particle of mass $\sim 60 \mathrm{GeV}$ from observed seasonal variation in the detector signal (originating, possibly, from a varying DM wind as the Earth traverses its path), subsequent experiments like CoGeNT [13], CRESST-II [14],

\footnotetext{
$\overline{1}$ While DM with ultra-suppressed electromagnetic couplings has been considered [10], such models are extremely ungainly and are unlikely to survive closer scrutiny.
} 
XENON100 [15], PandaX-II [16,17] and LUX [18] have not validated this; rather, they have only served to impose bounds in the plane described by the DM particle mass and its coupling to nucleons. Indirect-detection experiments, largely satellite-based, depend on the annihilation of a pair of DM particles into SM particles which are, subsequently, detected. Although there, occasionally, have been claims of anomalies in the data, unfortunately the experiments have failed to validate each other's positive sightings, resulting, once again, in further constraints [20-22]. Finally, we have the collider experiments, wherein excesses (over the SM expectations) in final states with large missing momentum are looked for. It should be realised, though, that even if such an excess is established, a DM explanation would still only be an hypothesis, for the only statement that can be made with certainty is that the produced neutral and colour-singlet particle is stable over the detector dimensions.

It is the last mentioned approach that we assume in this paper. While an investigation of the nature of the DM particles needs an understanding of the underlying physics, we adopt, instead, a model independent approach. Eschewing the details of the underlying dynamics, we begin by postulating a fermionic DM particle, and consider four-fermion operators involving these and the SM fermions. The relative strengths of these operators would, of course, be determined by the underlying dynamics. We assume that the operators involving quark currents are sub-dominant, as could happen, for example, if the dynamics, at a more fundamental level, involved a leptophilic boson. This immediately negates the constraints from the direct-detection experiments [15-18] (as the dominant interactions therein are with the nucleons) as also bounds from the LHC [23-37].

Such an assumption also alters the conclusions (on the interrelationship between the DM mass and the coupling strength) drawn from the deduced relic density. Starting with the Boltzmann equations describing the evolution of the particle densities, we derive the constraints on the same. As for the search strategies at a linear collider, the attention of the community, so far, has been largely commandeered by the final state comprising a single photon accompanied by missing energy [38-41]. While this continues to be an important channel, we augment this by other channels that are nearly as sensitive. Furthermore, we consider some novel effects of beam polarisation.

\section{Fermionic dark matter : a mini review}

As we have already mentioned, rather than considering the intricacies of particular models, we adopt the more conservative yet powerful concept of an effective field theory (EFT). Assuming that we have a single-component DM in the shape of a Dirac fermion $\chi$, we consider the least-suppressed (namely, dimension-six) operators involving a $\chi$-current and a SM fermion-current ${ }^{2}$. This simplifying assumption rules out the possibility of resonances and co-annihilations, significantly affecting the relic abundance of dark matter particles [42]. Furthermore, to reduce the number of possible operators, as also not to induce flavour changing neutral current processes, we restrict ourselves to only flavour-diagonal currents. A convenient parametrisation of such operators, for a single SM fermion $\psi$, is given by

$$
\begin{array}{ll}
\mathcal{O}_{V V}=\bar{\chi} \gamma_{\mu} \chi \bar{\psi} \gamma^{\mu} \psi, & \mathcal{O}_{A A}=\bar{\chi} \gamma_{\mu} \gamma_{5} \chi \bar{\psi} \gamma^{\mu} \gamma^{5} \psi, \\
\mathcal{O}_{S S}=\bar{\chi} \chi \bar{\psi} \psi, & \mathcal{O}_{P P}=\bar{\chi} \gamma_{5} \chi \bar{\psi} \gamma_{5} \psi, \\
\mathcal{O}_{V A}=\bar{\chi} \gamma_{\mu} \chi \bar{\psi} \gamma^{\mu} \gamma^{5} \psi, & \mathcal{O}_{A V}=\bar{\chi} \gamma_{\mu} \gamma_{5} \chi \bar{\psi} \gamma^{\mu} \psi, \\
\mathcal{O}_{S P}=\bar{\chi} \chi \bar{\psi} i \gamma_{5} \psi, & \mathcal{O}_{P S}=\bar{\chi} i \gamma_{5} \chi \bar{\psi} \psi,
\end{array}
$$

with the subscripts on the operators reflecting the Lorentz structure. The full interaction Lagrangian involving $\chi$ then can be parametrised as

$$
\begin{aligned}
\mathcal{L}_{\text {int }} & =\sum_{f} \sum_{M N \in \text { Ops. }} \frac{\alpha_{M N}^{f}}{\Lambda^{2}} \mathcal{O}_{M N}^{f} \\
& =\sum_{f} \sum_{M N \in \text { Ops. }}\left(\frac{g_{M N}^{f}}{\Lambda_{\text {Eff. }}}\right)^{2} \mathcal{O}_{M N}^{f} ; \Lambda_{\text {Eff. }}=\sqrt{4 \pi} \Lambda,
\end{aligned}
$$

where $\mathcal{O}_{M N}^{f} \equiv\left(\bar{\chi} \Gamma_{M} \chi\right)\left(\bar{f} \Gamma_{N} f\right)$ is a typical operator amongst those listed in (1), $\alpha_{M N}^{f}$ is the strength of the interaction and $\left|g_{M N}^{f}\right|<\sqrt{4 \pi}$ the corresponding coupling. $\Lambda_{\text {Eff. }}$. refers to the cut-off scale of the effective theory.

Before we proceed with our analysis, it is useful to consider the DM pair-annihilation cross section engendered by these operators [43]. Restricting ourselves to a single species ${ }^{3}$ $f$, and denoting the dimensionless $\zeta_{i} \equiv 2 m_{i}^{2} / s$, we have

$$
\begin{aligned}
\sigma_{V V}^{\mathrm{ann}} & =\frac{\left(\alpha_{V V}^{f}\right)^{2} N_{c}}{12 \pi \Lambda^{4}} \frac{\beta_{f}}{\beta_{\chi}} s\left(1+\zeta_{\chi}\right)\left(1+\zeta_{f}\right) \\
\sigma_{A A}^{\mathrm{ann}} & =\frac{\left(\alpha_{A A}^{f}\right)^{2} N_{c}}{12 \pi \Lambda^{4}} \frac{\beta_{f}}{\beta_{\chi}} s\left[1-2\left(\zeta_{\chi}+\zeta_{f}\right)+7 \zeta_{\chi} \zeta_{f}\right] \\
\sigma_{V A}^{\mathrm{ann}} & =\frac{\left(\alpha_{V A}^{f}\right)^{2} N_{c}}{12 \pi \Lambda^{4}} \frac{\beta_{f}^{3}}{\beta_{\chi}} s\left(1+\zeta_{\chi}\right) \\
\sigma_{A V}^{\mathrm{ann}} & =\frac{\left(\alpha_{A V}^{f}\right)^{2} N_{c}}{12 \pi \Lambda^{4}} \beta_{f} \beta_{\chi} s\left(1+\zeta_{f}\right)
\end{aligned}
$$

\footnotetext{
${ }^{2}$ We do not consider operators involving the SM bosons as they play only a subservient role in both direct and indirect detection.

${ }^{3}$ For multiple fermions, the cross sections, of course, add incoherently.
} 
$\sigma_{S S}^{\mathrm{ann}}=\frac{\left(\alpha_{S S}^{f}\right)^{2} N_{c}}{16 \pi \Lambda^{4}} \beta_{f}^{3} \beta_{\chi} s$

$\sigma_{P P}^{\mathrm{ann}}=\frac{\left(\alpha_{P P}^{f}\right)^{2} N_{c}}{16 \pi \Lambda^{4}} \frac{\beta_{f}}{\beta_{\chi}} s$

$\sigma_{S P}^{\mathrm{ann}}=\frac{\left(\alpha_{S P}^{f}\right)^{2} N_{c}}{16 \pi \Lambda^{4}} \beta_{f} \beta_{\chi} s$

$\sigma_{P S}^{\mathrm{ann}}=\frac{\left(\alpha_{P S}^{f}\right)^{2} N_{c}}{16 \pi \Lambda^{4}} \frac{\beta_{f}^{3}}{\beta_{\chi}} s$,

where $N_{c}=1$ (3) for leptons (quarks) and $\beta_{\chi, f}$ are the centre-of-mass frame velocities of $\chi(f)$ with $\beta_{i}=$ $\sqrt{1-2 \zeta_{i}}$. Clearly, for non-relativistic DM particles, $\sigma_{S S}^{\text {ann }}$ and $\sigma_{S P}^{\text {ann }}$ are the smallest. Similarly, for $m_{\chi} \gg m_{f}$ (as is the case except when $f$ is the top-quark), we have

$\sigma_{V A}^{\mathrm{ann}} \approx \sigma_{V V}^{\mathrm{ann}}, \quad \sigma_{A V}^{\mathrm{ann}} \approx \sigma_{A A}^{\mathrm{ann}}, \quad \sigma_{P P}^{\mathrm{ann}} \approx \sigma_{P S}^{\mathrm{ann}}$

These approximations would prove to be of considerable use in further analysis.

Dark matter interactions, as exemplified by equation (1), have been well studied in the context of the LHC [23-32,3437]. Clearly, the LHC would be more sensitive to operators involving the quarks. In this paper, we would be primarily interested in the orthogonal scenario, namely one wherein the DM current couples primarily to the SM leptons. To this end, we will assume that $g_{M N}^{q}=0$ for all of the operators. As we shall see in the next section, this would render insensitive even the dedicated direct search experiments.

\section{Relic density of leptophilic dark matter}

Before we proceed further with our analysis, it behoves us to consider the existing constraints on such a DM candidate. This is best understood if we consider a single operator at a time, and, henceforth, we shall assume this to be so. In other words, all operators bar the one under discussion shall be switched off. As to the couplings $\alpha_{M N}^{f}$, we consider two cases $^{4}$, namely

- Leptophilic: Switching on of all DM-lepton couplings $\alpha_{M N}^{l} \sim 1$ for all leptons $l$, and

- Electrophilic: Switching on of DM-electron coupling $\alpha_{M N}^{e} \sim 1$ only.

\footnotetext{
${ }^{4}$ Note that such a normalisation of couplings is quite standard in effective field theories.
}

Our choice of unity coupling $\alpha_{M N}^{f} \sim 1$ along with equation (2) allows us to express

$\frac{1}{\Lambda^{2}}=\left(\frac{g_{M N}^{f}}{\Lambda_{\text {Eff. }}}\right)^{2} \Rightarrow \Lambda_{\text {Eff. }}=\left|g_{M N}^{f}\right| \Lambda<\sqrt{4 \pi} \Lambda$.

Concentrating first on the epoch where the DM $(\chi)$ has frozen out, let us consider their pair annihilation to produce two light particles $(\ell)$, which, we assume, is in complete equilibrium, viz. $n_{\ell}=n_{\ell_{\mathrm{eq}}}$. The Boltzmann equation gives us:

$a^{-3} \frac{\mathrm{d}}{\mathrm{d} t}\left(n_{\chi} a^{3}\right)=\langle\sigma v\rangle\left[n_{\chi_{\mathrm{eq}}}^{2}-n_{\chi}^{2}\right]$

where $a \equiv a(t)$ is the scale factor and $\langle\cdots\rangle$ represents the thermal average. Here, $v$ is the relative velocity of a pair of $\chi$ 's, and $\sigma$ is the annihilation cross section.

In the radiation era, which we are interested in, the temperature scales as $a^{-1}$. We can, therefore, rewrite the Boltzmann equation, in terms of new dimensionless variables

$Y \equiv \frac{n_{\chi}}{T^{3}} \quad$ and $\quad x \equiv \frac{m_{\chi}}{T}$,

as

$\frac{\mathrm{d} Y}{\mathrm{~d} x}=-\frac{m_{\chi}^{3}\langle\sigma v\rangle}{H\left(m_{\chi}\right) x^{2}}\left(Y^{2}-Y_{\mathrm{eq}}^{2}\right)$

where $H\left(m_{\chi}\right)$ is the Hubble rate when the temperature equals $m_{\chi}$ and is given by

$H\left(m_{\chi}\right)=\sqrt{\frac{4 \pi^{3} G_{N} g_{\star}}{45}} m_{\chi}^{2}$.

Here $g_{\star}$ is the effective number of the degrees of freedom at that epoch.

To find the present density of DM particles, we need to find the solution of Eq. (7) in terms of the final freeze-out abundance, $Y_{\infty}$ (at $\left.x=\infty\right)$. While this, can be done only numerically, it is instructive to consider an approximate analytic solution. At early times $\left(T \gg m_{\chi}\right)$ reactions proceeded rapidly, and $n_{\chi}$ was close to its equilibrium value, $n_{\mathrm{eq}} \propto T^{3}$, or $Y \approx Y_{\text {eq }}$. As the temperature falls below $m_{\chi}$, the equilibrium abundance $Y_{\mathrm{eq}}=g /(2 \pi)^{3} x^{3 / 2} e^{-x}$ is exponentially suppressed. Consequently, the DM particles become so rare that they do not find each other fast enough to maintain the equilibrium abundance. This is the onset of freeze-out. In other words, well after freeze-out, $Y \gg Y_{\mathrm{eq}}$ and

$\frac{\mathrm{d} Y}{\mathrm{~d} x}=-\frac{m_{\chi}^{3}\langle\sigma v\rangle}{H\left(m_{\chi}\right) x^{2}} Y^{2}$ 
Integrating Eq. (9) from the freeze-out temperature $x_{f}$ until very late times $x=\infty$, we get

$$
Y_{\infty} \simeq \frac{x_{f} H\left(m_{\chi}\right)}{m_{\chi}^{3}\langle\sigma v\rangle} .
$$

The energy density, for the DM, which now is nonrelativistic, $\rho_{\chi}=m_{\chi} n_{\chi}$, falls as $a^{-3}$. The number density $n\left(a\left(T_{i}\right), T_{i}\right)$ in this post freeze-out epoch at any given temperature $T_{i}$ behaves as $Y_{\infty} T_{i}^{3}$. The number of the degrees of freedom also changes with the temperature from $g_{f}\left(x_{f}\right)$ at the freezing epoch to that of today is $g_{0}\left(x_{0}\right) \sim 3.36$ at the present day temperature $T_{0}$. The present energy density of DM can, thus, be re-written as

$\rho_{\chi}=m_{\chi} Y_{\infty} T_{0}^{3}\left(\frac{g_{0}\left(x_{0}\right)}{g_{f}\left(x_{f}\right)}\right)$.

It is customary to parametrise $\rho_{\chi} \equiv \Omega_{\chi} h^{2} \rho_{c}$, where $\rho_{c}=$ $1.05375 \times 10^{-5} h^{2}\left(\mathrm{GeV} / \mathrm{c}^{2}\right) \mathrm{cm}^{-3}$ is the critical density of the universe and the Hubble constant today being expressed as $H_{0}=h \times 100 \mathrm{~km} \mathrm{~s}^{-1} \mathrm{Mpc}^{-1}$. We then have

$$
\Omega_{\chi} h^{2}=\sqrt{\frac{4 \pi^{3} G_{N} g_{\star}\left(x_{f}\right)}{45}} \frac{x_{f} T_{0}^{3} g_{0}}{\rho_{c}\langle\sigma v\rangle g_{\star}\left(x_{f}\right)} .
$$

Using $T_{0}=2.72548 \pm 0.00057 \mathrm{~K}$ [44], the contribution of the Dirac fermion $\chi$, with its two degrees of freedom, is

$\Omega_{\mathrm{DM}}^{(\chi)} h^{2} \approx 2 \times x_{f} \frac{1.04 \times 10^{9} \mathrm{GeV}^{-1}}{m_{p l} \sqrt{g_{\star}\left(x_{f}\right)}\langle\sigma v\rangle}$.

While the WMAP 9-year data gives $\Omega_{\mathrm{DM}} h^{2}=0.1138 \pm$ 0.0045 , the Planck 2015 results [8] suggest a marginally different value, namely $\Omega_{\mathrm{DM}} h^{2}=0.1199 \pm 0.0022$. This translates to a strict relation in the mass-coupling plane for $\chi$. However, a conservative assumption would be that the relic density of the $\chi$ does not saturate the observed DM energy density, or $\Omega_{\chi}<\Omega_{\mathrm{DM}}$. This would impose a lower bound on the self-annihilation cross section for the $\chi$, or, in other words, an upper bound on $\Lambda$.

\subsection{The annihilation cross sections}

In the calculation of the probability of a DM particle being annihilated by another one, it is useful to consider the rest frame of the first and re-express the aforementioned annihilation cross sections in terms of the velocity $v$ of the second particle in his frame. Clearly, $v=\left(2 \beta_{\chi}\right) /\left(1+2 \beta_{\chi}^{2}\right)$ and, working with a small $v$ expansion (relevant for nonrelativistic DM), we have $\beta_{\chi} \simeq \frac{v}{2}\left[1+\frac{v^{2}}{4}+\frac{v^{4}}{8}\right]+\mathcal{O}\left(v^{7}\right)$ and $s \simeq m_{\chi}^{2}\left[4+v^{2}+\frac{3}{4} v^{4}\right]+\mathcal{O}\left(v^{6}\right)$. The corresponding $v$-expansions, to $\mathcal{O}\left(v^{2}\right)$, are

$$
\begin{aligned}
& \sigma_{S S}^{\mathrm{ann}} v \simeq \frac{\left(\alpha_{S S}^{l}\right)^{2} N_{c}}{8 \pi \Lambda^{4}}\left(1-\xi_{f}\right)^{3 / 2} m_{\chi}^{2} v^{2} \\
& \sigma_{P P}^{\mathrm{ann}} v \simeq \frac{\left(\alpha_{P P}^{l}\right)^{2} N_{c}}{2 \pi \Lambda^{4}} \sqrt{1-\xi_{f}} m_{\chi}^{2}\left[1+\frac{\xi_{f} v^{2}}{8\left(1-\xi_{f}\right)}\right]
\end{aligned}
$$$$
\sigma_{V V}^{\mathrm{ann}} v \simeq \frac{\left(\alpha_{V V}^{l}\right)^{2} N_{c}}{2 \pi \Lambda^{4}} \sqrt{1-\xi_{f}}\left(2 m_{\chi}^{2}+m_{f}^{2}\right)
$$$$
\left[1+\frac{-4+2 \xi_{f}+11 \xi_{f}^{2}}{24\left(1-\xi_{f}\right)\left(2+\xi_{f}\right)} v^{2}\right]
$$

$\sigma_{A A}^{\mathrm{ann}} v \simeq \frac{\left(\alpha_{A A}^{l}\right)^{2} N_{c}}{2 \pi \Lambda^{4}} \sqrt{1-\xi_{f}} m_{f}^{2}$

$$
\left[1+\frac{8 \xi_{f}^{-1}-28+23 \xi_{f}}{24\left(1-\xi_{f}\right)} v^{2}\right]
$$

where $\xi_{f} \equiv m_{f}^{2} / m_{\chi}^{2}$. Clearly, the $v$-independent terms emanate from $s$-wave scattering alone, while the $\mathcal{O}\left(v^{2}\right)$ piece receives contributions from both $s$-wave and $p$-wave annihilation processes.

The quantity of interest is not just $\sigma^{\text {ann }}$, but the thermal average $\left\langle\sigma_{M N}^{\mathrm{ann}} v\right\rangle$, since this provides a measure of the rate at which a DM particle will meet another with the appropriate velocity and get annihilated. Assuming $\chi \chi \rightarrow e^{+} e$ to be the dominant channel into which the $\chi$ annihilate, we can estimate the thermally averaged value of $\sigma(\chi \chi \rightarrow$ $\left.e^{+} e^{-}\right) v$.

The inferred value of $\Omega_{\chi} h^{2}$, when ascribed to WIMPs with a mass of a few hundred GeVs, requires $\langle\sigma(\chi \chi \rightarrow$ $\left.\left.e^{+} e^{-}\right) v\right\rangle \approx 3 \times 10^{-26} \mathrm{~cm}^{3} \mathrm{~s}^{-1}$. Taking a cue from this, we analyse upper bounds on $\left\langle\sigma\left(\chi \chi \rightarrow e^{+} e^{-}\right) v\right\rangle$ in Sect. 5 from the sensitivity study of the DM signatures from ILC for the kinetically accessible DM mass range.

In Fig. 1 we present the relic density plots for the interactions parametrised by the operators mentioned in Sect. 2 corresponding to the leptophilic and electrophilic scenarios. We implement the model containing effective interactions of DM particle with the SM sector using FeynRules [46] and generate the model files for CalcHEP, which is required for the relic density calculation in micrOmegas [47]. These results have been verified with MadDM $[48,49]$.

The curves in Fig. 1 imply that the correct amount of energy density requires the mass of dark matter particles to increase with $\Lambda$. We can infer this behaviour from (13) as $\Omega_{\chi} h^{2}$ mainly depends on $\langle\sigma v\rangle$. From Eqs. (14a)(14d) we see that the thermal average in equation (13) i.e. $\langle\sigma v\rangle$ is proportional to $m_{\chi}^{2} / \Lambda^{4}$ for $m_{\chi}$ values greater than $\sim 10 \mathrm{GeV}$. This implies that, for a fixed value of $\Omega_{\chi} h^{2}, \Lambda^{4} / m_{\chi}^{2}$ is a constant. This, in turn, translates to $\Lambda \propto \sqrt{m_{\chi}}$. 


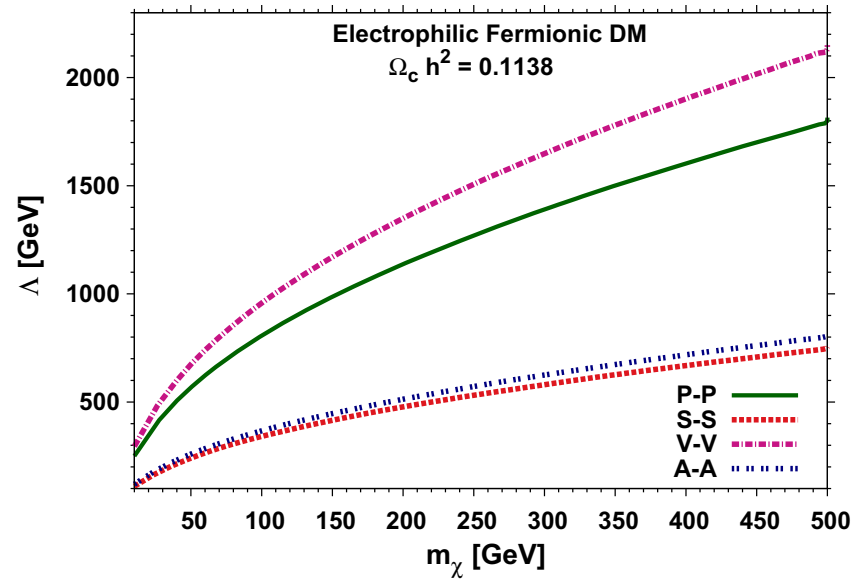

Fig. 1 Contours in the $\Lambda-m_{\chi}$ plane based on the cold dark matter density $\Omega_{c} h^{2}=0.1138 \pm 0.0045$ obtained from the 9-year WMAP data [45]. Here we have taken $\alpha_{P P}^{l}=\alpha_{S S}^{l}=\alpha_{V V}^{l}=\alpha_{V A}^{l}=1$. These curves

\section{Direct and indirect detection}

\subsection{DM-electron scattering}

We now turn to direct-detection search, where both electrophilic and leptophilic DM are scattered by:

(a) the bound electron of an atom and hence the recoiled electron is ejected out of the atom,

(b) the bound electron and the recoil is taken by the atom as a whole and

(c) the quarks, where the DM is attached to the loop of charged virtual leptons which in turn interacts with the quarks via photon exchange and hence the nucleus recoil is measured in the detector.

In this article we restrict our study for the DM-electron scattering only. We analyse the free electron scattering cross sections with the DM for the scalar, pseudo-scalar, vector and axial couplings, respectively, and are given as

$\sigma_{\chi e}^{S S}=\frac{\left(\alpha_{S S}^{l}\right)^{2} m_{e}^{2}}{\pi \Lambda^{4}}$

$\sigma_{\chi e}^{P P}=\frac{\left(\alpha_{P P}^{l}\right)^{2} m_{e}^{2}}{3 \pi \Lambda^{4}} \frac{m_{e}^{2}}{m_{\chi}^{2}} v^{4}$,

$\sigma_{\chi e}^{V V}=\frac{\left(\alpha_{V V}^{l}\right)^{2} m_{e}^{2}}{\pi \Lambda^{4}}$

$\sigma_{\chi e}^{A A}=\frac{3\left(\alpha_{A A}^{l}\right)^{2} m_{e}^{2}}{\pi \Lambda^{4}}$

where $v \sim 10^{-3} c$, is the DM velocity in our halo. We observe that the cross section with the axial-vector couplings of DM dominates over all others. The pseudo-scalar type coupling is

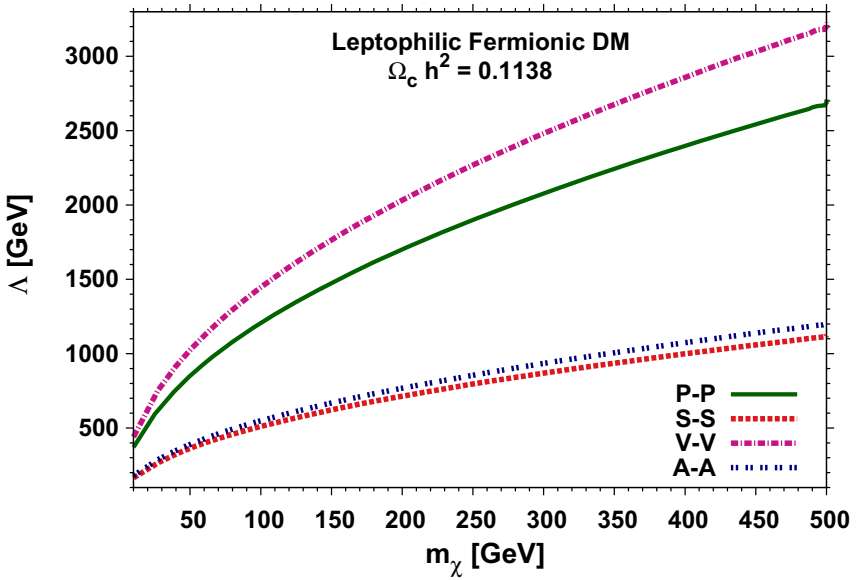

are obtained using micrOmegas-v-3.5.5 [47], the regions below the curves are allowed by the relic density bounds

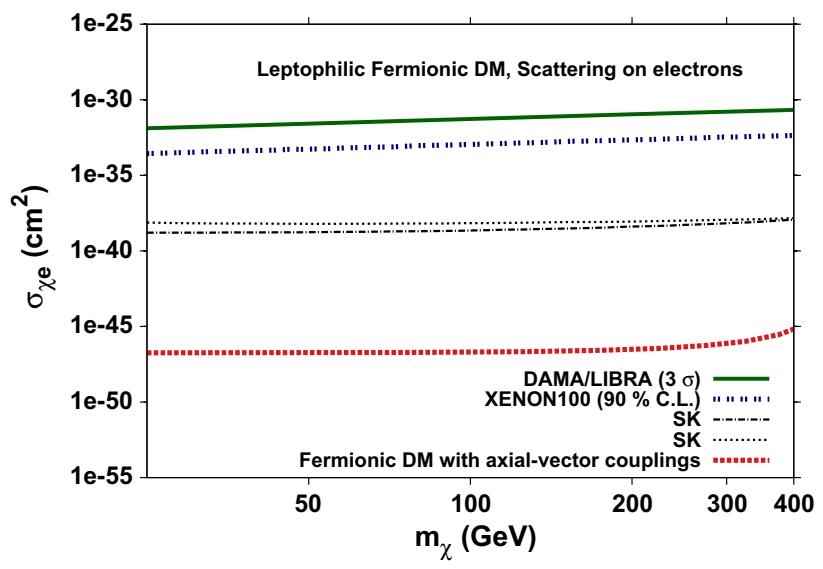

Fig. 2 The scattering cross section of DM on a free electron with the axial-vector interactions $\sigma\left(\chi e^{-} \rightarrow \chi e^{-}\right)$is depicted for the DM mass varying between 25 and $400 \mathrm{GeV}$. The cross sections are computed using the upper limit on $\Lambda$ obtained from the relic density curves in Fig. 1 corresponding to the flavour-universal couplings for DM with leptons $\alpha_{A A}^{l}=1$. Exclusion plots from DAMA at $90 \%$ and $3 \sigma$ C.L. for the case of DM-electron scattering are also shown [50]. Bounds at $90 \%$ C.L. are shown for XENON100 from inelastic DM-atom scattering [51]. The dashed curves show the $90 \%$ CL constraint from the SuperKamiokande limit on neutrinos from the Sun, by assuming annihilation into $\tau^{+} \tau^{-}$or $\nu \bar{v}[50]$

highly suppressed due to its dependence on the fourth power of the electron mass and velocity. Respective cross sections for the case of bound electrons are likely to be enhanced w.r.t. that of free electrons nearly by a factor of $\sim 10^{5}$ [50]. Using the constraints from the relic density on the lower bound of the couplings for a given DM mass $m_{\chi}$, in Fig. 2, we present the scattering cross section of the DM with free electrons via the most dominant channel with axial-vector couplings. We compare our result with the recent $3 \sigma$ limits from the DAMA/ LIBRA experiment [50] and 90\% C.L. data from XENON100 [51]. However, even with the inclusion of the 


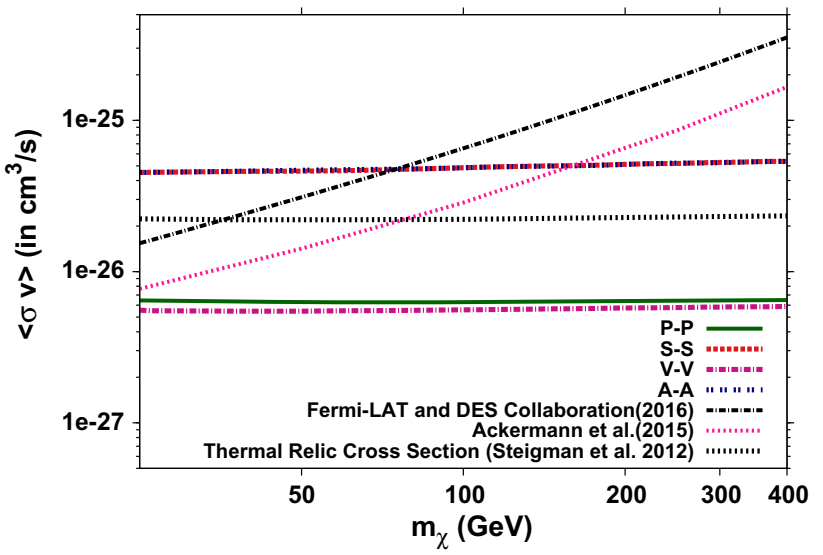

Fig. 3 The variation of the thermal averaged DM annihilation cross sections versus the leptophilic DM mass $m_{\chi}$. The cross sections are computed using the upper limit on $\Lambda$ obtained from the relic density curves in Fig. 1 corresponding to the flavour-universal couplings for $\mathrm{DM}$ with leptons $\alpha_{P P}^{l}=\alpha_{S S}^{l}=\alpha_{V V}^{l}=\alpha_{A A}^{l}=1$. These are compared with the median of the thermal averaged DM annihilation cross section derived from a combined analysis of the nominal target sample for the $\tau^{+} \tau^{-}$channel assuming a $100 \%$ branching fraction $[19,20]$

correction factor arising from the the bounded electrons, the contributions of the axial-vector couplings of DM to the scattering cross section are far below these experimental upper bounds.

Indeed, the XENON100 collaboration has constrained the $\sigma(\chi e \rightarrow \chi e) \lesssim 10 \mathrm{pb}$ at $90 \%$ C.L. for ${ }^{5} 0.6 \mathrm{GeV}<m_{\chi}<$ $1 \mathrm{TeV}$ [51] for the case of axial-vector coupling. Expectedly, these constraints are much stronger (by nearly an order of magnitude) than the cross sections required to explain the DAMA/LIBRA results [12]. Interestingly, the strongest bounds emanate from solar physics. In general, the neutrino flux coming from DM annihilations inside the sun is proportional to the DM scattering cross section. But, working in the region where DM capture and its annihilations inside the star are in equilibrium makes the neutrino flux independent of the DM annihilation. As the DM particles can be trapped in the solar gravitational field, their annihilation into neutrinos would modify the solar neutrino spectrum and, consequently, the SuperKamiokande measurements [50]. This translates to $\sigma\left(\chi \bar{\chi} \rightarrow \tau^{+} \tau^{-}\right) \lesssim 0.1 \mathrm{pb}$ and $\sigma(\chi \bar{\chi} \rightarrow \nu \bar{\nu}) \lesssim 0.05 \mathrm{pb}$, which are relevant for the general leptophilic case with axialvector coupling. Understandably, no such bound exists for the purely electrophilic case.

\subsection{DM annihilation}

Next we discuss the bounds from the indirect-detection experiments, wherein we can directly use the cross sections given in Eqs. (3a)-(3h) and fold them with the local veloc-

\footnotetext{
5 The exclusion limits do have a dependence on $m_{\chi}$ with the maximum sensitivity occurring at $m_{\chi} \sim 2 \mathrm{GeV}$ and relaxing by an order of magnitude for the highest $m_{\chi}$ values.
}

ity of the DM particle. The latter, of course, would depend crucially on the particular profile of the DM distribution that one adopts.

From Eqs. (14a)-(14d), the thermally averaged annihilation cross section of a leptophilic DM of mass $m_{\chi}$ can be estimated using the upper bound on the $\Lambda$ for a fixed DM coupling (or lower bound on the scalar, pseudo-scalar, vector and axial-vector couplings of DM for a fixed cut-off 1 ) w.r.t. a given $m_{\chi}$ obtained from the relic density constraints. The annihilation cross section for the leptophilic DM is marginally higher than electrophilic DM and roughly are of the same order of magnitude for $m_{\chi} \sim 10-400 \mathrm{GeV}$. We compute the thermal averaged annihilation cross section $\chi \bar{\chi} \rightarrow \tau^{+} \tau^{-}$for the leptophilic DM with the upper bound on $\Lambda$ obtained from the relic density constraints for $\alpha_{P P}^{l}=\alpha_{S S}^{l}=\alpha_{V V}^{l}=\alpha_{A A}^{l}=1$ and depict in Fig. 3. Here we have assumed universal couplings of DM with all three generation of massive charged leptons.

In a recent article $[19,20], \mathrm{DM}$ annihilation cross-section constraints at $95 \% \mathrm{CL}$ for the $b \bar{b}$ and $\tau^{+} \tau^{-}$channels are derived from a combined analysis of $15 \mathrm{dSphs.} \mathrm{We} \mathrm{compare}$ our thermally averaged DM annihilation cross section for the $\tau^{+} \tau^{-}$channel in Fig. 3 with the one computed in this reference assuming $100 \%$ branching fraction for each of these channels.

Table 1 Accelerator parameters for each of the run scenarios considered in this paper. $P_{-}$and $P_{+}$represent the electron and positron polarisations, respectively

\begin{tabular}{lllll}
\hline & ILC-500 & ILC-500P & ILC-1000 & ILC-1000P \\
\hline$\sqrt{s}($ in GeV) & 500 & 500 & 1000 & 1000 \\
$L_{\text {int }}\left(\mathrm{in} \mathrm{fb}^{-1}\right)$ & 500 & 250 & 1000 & 500 \\
$P_{-}$ & 0 & $80 \%$ & 0 & $80 \%$ \\
$P_{+}$ & 0 & $30 \%$ & 0 & $30 \%$ \\
\hline
\end{tabular}

Table 2 The efficiency $S / \sqrt{(S+B)+\delta_{\text {sys }}^{2}(S+B)^{2}}$ with $\delta_{\text {sys }}=1 \%$ for the process $e^{+} e^{-} \rightarrow j j+E_{T}$ on imposition of the basic cuts for $\Lambda=1 \mathrm{TeV}$ corresponding to the three representative values of the DM mass with unpolarised and polarised initial beams. The respective coupling of DM with the charged lepton $\alpha_{M N}^{e}$ is taken to be unity

\begin{tabular}{llllll}
\hline & \multicolumn{3}{l}{ Unpolarised } & & Polarised \\
\cline { 2 - 3 } Interactions & $\mathrm{S}-\mathrm{S}$ & $\mathrm{V}-\mathrm{V}$ & & $\mathrm{S}-\mathrm{S}$ & $\mathrm{V}-\mathrm{V}$ \\
$\sqrt{s}$ in $\mathrm{TeV}$ & 1.0 & 1.0 & & 1.0 & 1.0 \\
$\mathcal{L}$ in $\mathrm{fb}^{-1}$ & 1000 & 1000 & & 500 & 500 \\
$\left(P_{e^{-}}, P_{e^{+}}\right)$ & $(0,0)$ & $(0,0)$ & & $(0.8,-0.3)$ & $(0.8,-0.3)$ \\
\hline $\mathrm{DM}$ mass in GeV & & & & \\
75 & 9.46 & 3.27 & 30.78 & 15.79 \\
225 & 3.76 & 2.93 & 14.34 & 14.31 \\
325 & 0.72 & 1.94 & 3.00 & 9.87 \\
\hline
\end{tabular}



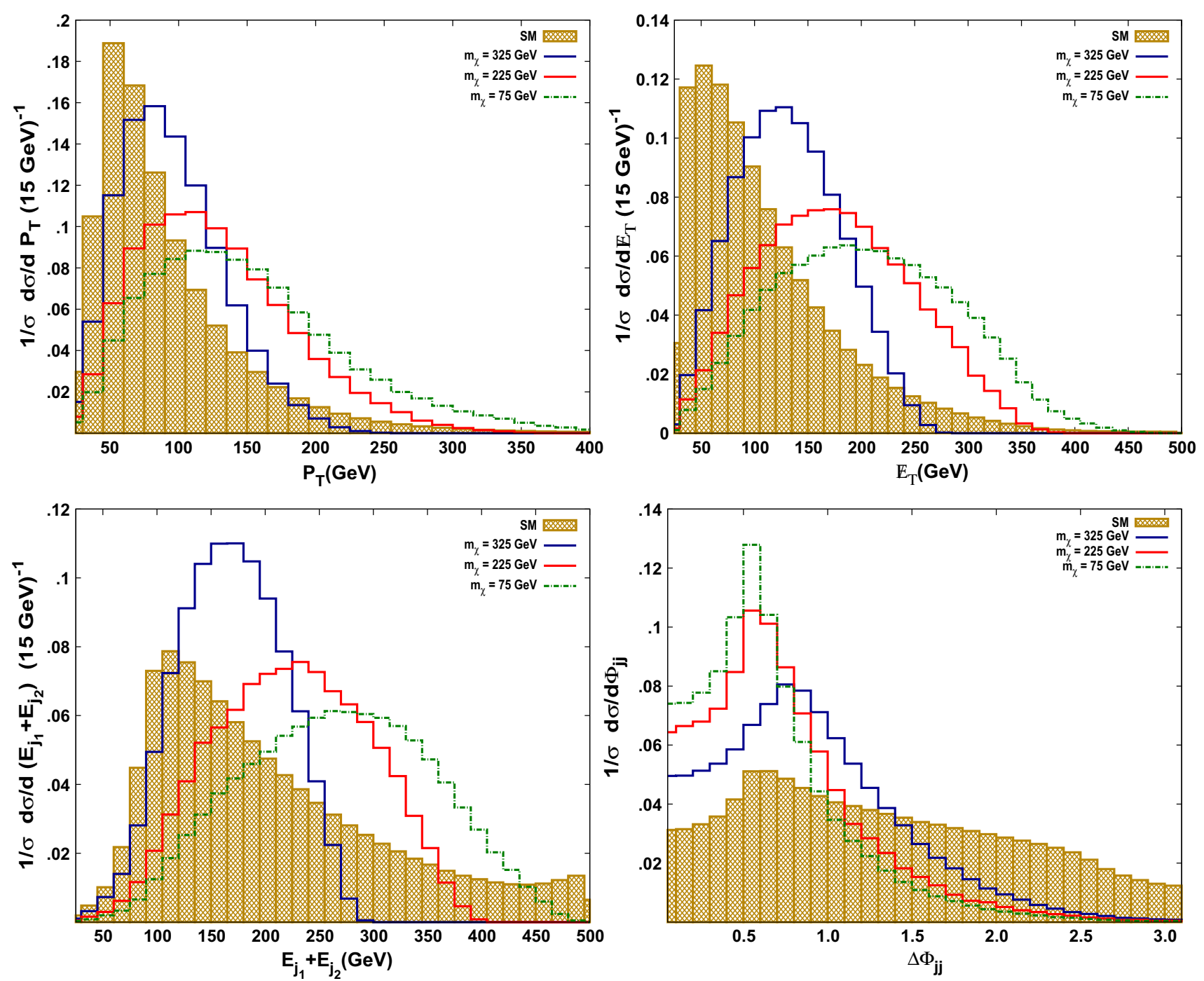

Fig. 4 Normalised 1-D differential distribution of the cross section w.r.t. kinematic observable $P_{T j_{1}}, E_{T}$, sum of energy of jet pairs and $\Delta \phi_{j j}$ for the background process (shaded histograms) and the $Z \rightarrow j j$ associated DM pair production at the three representative values of

\section{Interesting signatures at ILC}

While DM particles can be produced in many different processes at a given collider, only a few of them are, potentially, of interest. Remembering that the DM particle has to be produced only in pairs, and that there must be at least one visible particle in the final state so as to register the event in a detector, the simplest process at a linear collider is, of course, $e^{+} e^{-} \rightarrow \bar{\chi} \chi \gamma$, and it has, rightly, been well studied in many different contexts [38-40]. Although the signature, namely mono-photon with missing energy-momentum, can be masqueraded by both detector effects as well as a large and irreducible background emanating from $e^{+} e^{-} \rightarrow \bar{v}_{i} v_{i} \gamma$, the kinematic profiles are sufficiently different enough to merit the possibility of a discovery.

$M_{\chi}=75,225$ and $325 \mathrm{GeV}$ with $\sqrt{s}=1 \mathrm{TeV}, \Lambda=1 \mathrm{TeV}$ and $\alpha_{S S}^{l}=\alpha_{P P}^{l}=\alpha_{S P}^{l}=\alpha_{P S}^{l}=1$. The bin width is 0.1 for the $\Delta \phi_{i i}$ distribution, while it is $15 \mathrm{GeV}$ for the $E_{T}$ and $E_{j_{1}}+E_{j_{2}}$

Given the exceeding simplicity of the aforementioned channel, attention has focused on it almost exclusively. However, it is worthwhile to explore complementary channels, like, DM pair production in association with on-shell $Z$ or off-shell $Z^{\star}$,

$e^{+} e^{-} \rightarrow \chi \bar{\chi}+Z\left(Z \rightarrow j j, \mu^{+} \mu^{-}, e^{+} e^{-}\right)$

where $j$ refers to a jet arising from any of $u, d, s, c, b$. In this paper, we shall not attempt to impose any $b$-tagging (or $b$-veto) requirements, and, hence, the $b$-jet is equivalent to any other light quark jet ${ }^{6}$. To obtain a realistic analysis, we

\footnotetext{
${ }^{6}$ While similar analyses have been attempted in Refs. [52,53], our conclusions differ substantially from their conclusions even when we adopt an approach identical to theirs. Furthermore, we here present a much more refined analysis.
} 

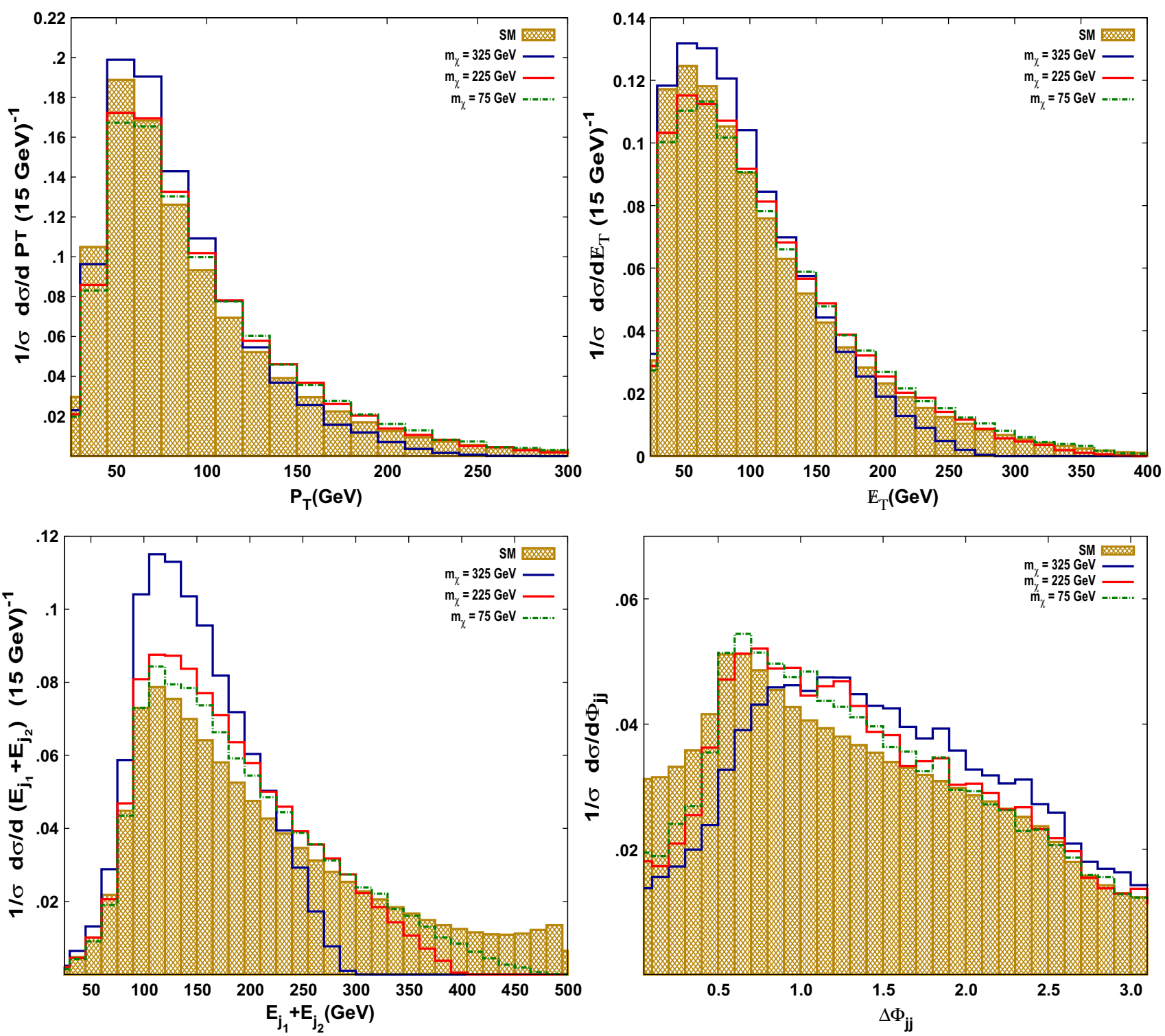

Fig. 5 Normalised 1-D differential distribution of the cross section w.r.t. kinematic observables $P_{T} j_{1}, / E_{T}$, sum of energy of jet pairs and $\Delta \phi_{j j}$ for the background process (shaded histograms) and the $Z \rightarrow j j$ associated DM pair production at the three representative

values of $M_{\chi}=75$ and $325 \mathrm{GeV}$ at $\sqrt{s}=1 \mathrm{TeV}, \Lambda=1 \mathrm{TeV}$ and $\alpha_{V V}^{l}=\alpha_{A A}^{l}=\alpha_{V A}^{l}=\alpha_{A V}^{l}=1$. The bin width is 0.1 for the $\Delta \phi_{i i}$ distribution, while it is $15 \mathrm{GeV}$ for the $E_{T}$ and $E_{j_{1}}+E_{j_{2}}$

$e^{+} e^{-} \rightarrow e^{+} e^{-}+\sum_{i} v_{i} \bar{\nu}_{i}$

thus, we need to consider $e^{+} e^{-} \rightarrow f \bar{f}+\bar{\chi} \chi$, where $f \equiv$ jet, $e^{-}, \mu^{-}$. The corresponding irreducible SM backgrounds emanate from $^{7}$

$e^{+} e^{-} \rightarrow 2$ jets $+\sum_{i} v_{i} \bar{v}_{i}$

$e^{+} e^{-} \rightarrow \mu^{+} \mu^{-}+\sum_{i} v_{i} \bar{\nu}_{i}$

\footnotetext{
7 In all our analysis, the dijet (plus missing energy) signal is actually an inclusive one, in that we demand a minimum of two jets.
}

respectively. The largest contribution to the first mode in Eq. (17a) accrues from $e^{+} e^{-} \rightarrow Z+\gamma^{*} / Z^{*}$ with $Z \rightarrow$ $\nu_{i} \bar{v}_{i}$ and off-shell photon or weak neutral gauge boson exchange $\gamma^{*} / Z^{*}$ splitting to two jets. This dominant process is then followed by the $W^{+} W^{-}$fusion channel $e^{+} e^{-} \rightarrow$ $v_{e} \bar{v}_{e} W^{+} W^{-} \rightarrow v_{e} \bar{v}_{e} j j$ and t-channel-like topologies $W$ exchange diagrams. Thus, we have considered all the gauge invariant connected Feynman diagrams at the tree level corresponding to the process $e^{+} e^{-} \rightarrow \chi \bar{\chi} j j$. 
Feynman diagrams analogous to both these sets also contribute to the two other processes given in (17b) and (17c) respectively. The relative strengths of these channels depend on a multitude of factors, such as the centre-of mass energy, beam polarisation (if any) and the kinematical cuts imposed.

We use MadGraph5 [54] to perform the simulations for both SM background and the signal. We employ PYTHIA6 [55] to carry out the parton shower and hadronisation. PGS [56] is used for Fast detector simulation with anti- $k_{T}$ algorithm for jet reconstruction. In accordance with technical design report for ILC detectors [57], we set the energy smearing parameters of the electromagnetic calorimeter and the hadronic calorimeter in the PGS card as

$$
\begin{aligned}
\frac{\Delta E}{E} & =\frac{17 \%}{\sqrt{E / \mathrm{GeV}}} \oplus 1 \%, \\
\frac{\Delta E}{E} & =\frac{30 \%}{\sqrt{E / \mathrm{GeV}}} .
\end{aligned}
$$

The other accelerator parameters used in our analysis are also set according to the technical design report for ILC [58] and we tabulate them in Table 1 for convenience.

Before we commence an analysis of the cross sections and the kinematic distributions we must impose basic (acceptance) cuts on transverse momenta $\left(p_{T j / \ell}\right)$, rapidities $\left(\eta_{j / \ell}\right)$ and separation $\left(\Delta R_{j j / \ell \ell}\right)$ of the visible entities (jets and $\mu^{ \pm}$, $e^{ \pm}$) along with the missing transverse energy $\left(E_{T}\right)$, which are commensurate with the typical detector capabilities on the one hand, and with theoretical considerations (such as jet definitions) on the other.

- $p_{T_{i}} \geq 10 \mathrm{GeV}$ where $i=\ell, j$,

- $\left|\eta_{\ell}\right| \leq 2.5$ and $\left|\eta_{j}\right| \leq 5$,

- $\Delta R_{i i} \geq 0.4$ where $i=\ell, j$,

- $\left|M_{i i}-m_{Z}\right| \leq 5 \Gamma_{Z}$ where $i=\ell, j$; for the hadronic channel, this invariant mass actually refers to that for all the jets together,

- $E_{T} \geq 25 \mathrm{GeV}$.

The corresponding cross sections with these basic cuts for the polarised and unpolarised beams of electron and positron are summarised in Table 2. Feynman diagrams consisting of different topologies and contributing to the background (17a) can be further suppressed by imposing the most-dominant invariant-mass cut of the visible particles ${ }^{8}$, and thus allowing $Z+Z^{(*)}$ final state with the on-shell $Z$ decaying into the two visible entities, and the off-shell $Z^{(*)}$ going to a neutrino pair. Within this approximation, the diagrams contributing

\footnotetext{
$\overline{{ }^{8} \text { For } e^{+} e^{-} \rightarrow} e^{+} e^{-}+\sum_{i} \nu_{i} \bar{\nu}_{i}$, the deviation from such a dominance is quite significant. Precisely for this reason, this turns out to be to be the least sensitive of the three channels.
}

to the SM background processes (17a)-(17c) have identical topologies and differ only in the coupling of the $Z$ to the final state fermions. Consequently, this difference would manifest itself essentially only in the total rates with differences in the normalised distributions being of a sub-leading nature. This is true even for the signal events corresponding to these final states. Hence we have displayed the one-dimensional normalised differential cross section for process with only jets as final state in Figs. 4 and 5.

\subsection{Analysis with one- and two-dimensional distributions:-}

In order to augment the signal-to-noise ratio by imposing additional selection cuts on the kinematic observables, we recourse ourselves to the detailed study of the normalised one-dimensional differential cross-section distributions w.r.t. for all the sensitive kinematic observables e.g. $p_{T_{j / l}}, E / t_{T}, \Delta \phi_{i i}$ and sum of energies from a jet or lepton pair $\left(E_{i_{1}}+E_{i_{2}}\right)$ for the signal with varying DM mass as well as varying effective DM pair - SM fermion pair couplings $\alpha_{M N}^{f} / \Lambda^{2}$ at $\sqrt{s}=500$ $\mathrm{GeV}$ and $1 \mathrm{TeV}$.

The very structure of the DM interaction Lagrangian implies that, for a given final state, the normalised differential distributions for the SS, SP, PS and PP cases are very similar to each other, with the differences being proportional to the difference in mass of the SM fermions in the final state (i.e., $e, \mu$ or the light quarks). Similarly, the normalised distributions for the VV, VA, AV and the AA cases are, again, very similar to each other. Therefore, we have chosen to display the normalised histograms for the observables $p_{T_{j / l}}$, $E /_{T}, \Delta \phi_{i i}$ and $\left(E_{j_{1}}+E_{j_{2}}\right)$ from signal processes at the three representative values of the DM masses $m_{\chi}$ namely 75, 225 and $325 \mathrm{GeV}$ in Figs. 4 and 5 corresponding to the scalar and vector interactions of the DM bilinears with SM fermion pair at $\sqrt{s}=1 \mathrm{TeV}$ and $\Lambda=1 \mathrm{TeV}$. We list our observations as follows:

- In Fig. 4, we observe that, as we increase the DM mass, the respective shape profile of all the kinematic observables become more and more distinct w.r.t. the SM contribution. This difference in shape profile for the scalar coupling with that of SM can be attributed to the chiralityflip interactions of the DM which becomes much more pronounced for higher values of $m_{\chi}$.

On contrary, the shape profiles of all the kinematic distributions with vector coupling of DM in Fig. 5, behave the same as those of $\mathrm{SM} Z \rightarrow v \bar{v}$ coupling.

- We find that in the lower $m_{\chi}$ region, the jet pair remains highly boosted as in the case of SM and hence, the peak of the differential cross-section distribution w.r.t. $\Delta \phi_{j j}$ coincides with that of SM background around $35^{\circ}$. The peak, however, shifts towards higher values of $\Delta \phi_{j j}$ as we increase $m_{\chi}$. 

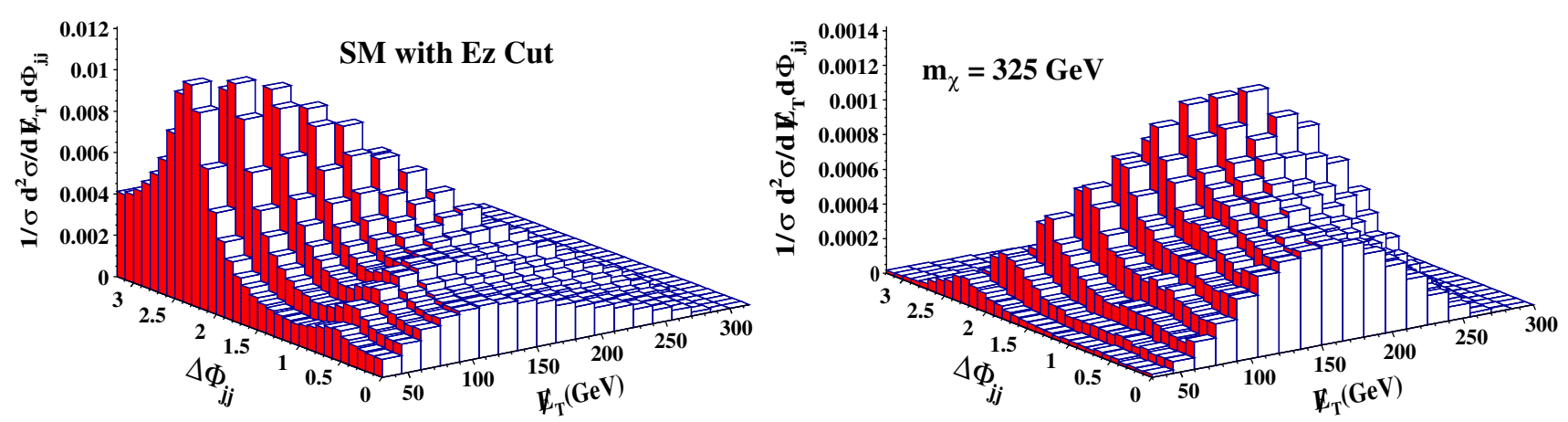

Fig. 6 Lego plots showing normalised 2-D differential cross-section distribution w.r.t. $\not_{T}$ and $\Delta \phi_{j j}$ for the background and the $Z \rightarrow j j$ associated DM pair production for $M_{\chi}=325 \mathrm{GeV}$ at $\sqrt{s}=1 \mathrm{TeV}, \Lambda=1 \mathrm{TeV}$ and $\alpha_{S S}^{l}=1$. The bin width is $15 \mathrm{GeV}$ for the $\mathbb{E}_{T}$ and 0.1 for the $\Delta \phi_{i i}$

Table 3 Estimation of $3 \sigma$ sensitivity reach of the maximum value of the cut-off $\Lambda_{\text {max. }}$ in TeV for $m_{\chi}=20 \mathrm{GeV}$ that can be probed in ILC at $\sqrt{s}$ $=500 \mathrm{GeV}$ and $1 \mathrm{TeV}$ with an integrated luminosity $\mathcal{L}=500 \mathrm{fb}^{-1}$ and $\mathcal{L}$
$=1 \mathrm{ab}^{-1}$ respectively, through all visible channels of $e^{+} e^{-} \rightarrow Z+\chi \bar{\chi}$. The respective coupling of DM with the charged lepton $\alpha_{M N}^{l}$ is taken to be unity

\begin{tabular}{|c|c|c|c|c|c|c|c|c|}
\hline \multirow[b]{2}{*}{$\begin{array}{l}\text { Interactions } \\
\sqrt{s} \text { in } \mathrm{TeV} \\
\mathcal{L} \text { in } \mathrm{fb}^{-1} \\
\left(P_{e^{-}}, P_{e^{+}}\right)\end{array}$} & \multicolumn{4}{|c|}{ Unpolarised } & \multicolumn{4}{|l|}{ Polarised } \\
\hline & $\begin{array}{l}\text { S-S } \\
0.5 \\
500 \\
(0,0)\end{array}$ & $\begin{array}{l}1 \\
1000 \\
(0,0)\end{array}$ & $\begin{array}{l}\mathrm{V}-\mathrm{V} \\
0.5 \\
500 \\
(0,0)\end{array}$ & $\begin{array}{l}1 \\
1000 \\
(0,0)\end{array}$ & $\begin{array}{l}S-S \\
0.5 \\
250 \\
(0.8,-0.3)\end{array}$ & $\begin{array}{l}1 \\
500 \\
(0.8,-0.3)\end{array}$ & $\begin{array}{l}\mathrm{V}-\mathrm{V} \\
0.5 \\
250 \\
(0.8,0.3)\end{array}$ & $\begin{array}{l}1 \\
500 \\
(0.8,0.3)\end{array}$ \\
\hline$e^{+} e^{-} \rightarrow \mu^{+} \mu^{-}+\not E_{T}$ & 0.67 & 1.74 & 0.55 & 1.05 & 0.7 & 2.02 & 0.6 & 1.18 \\
\hline$e^{+} e^{-} \rightarrow e^{+} e^{-}+E_{T}$ & 0.62 & 1.64 & 0.52 & 1 & 0.63 & 1.67 & 0.55 & 1.15 \\
\hline$e^{+} e^{-} \rightarrow j j+\not E_{T}$ & 0.98 & 2.62 & 0.81 & 1.53 & 1.1 & 2.87 & 0.89 & 1.88 \\
\hline$e^{+} e^{-} \rightarrow Z+\not E_{T}$ & 0.99 & 2.64 & 0.82 & 1.55 & 1.1 & 2.88 & 0.89 & 1.89 \\
\hline
\end{tabular}

- We find that the energy profile of the visible pair $E_{j_{1}}+E_{j_{2}}$ are sensitive to the choice of the DM mass and thus advocating that the upper limit on this kinematic observable can be used as a dynamic mass dependent selection cut to reduce the continuum background. This is realised by invoking a cut on the invariant mass of visible particles to be around $\left|\left(p_{j_{1}}+p_{j_{2}}\right)^{2}-m_{Z}^{2}\right|^{\frac{1}{2}} \leq 5 \Gamma_{Z}$, where $\Gamma_{Z}=2.49 \mathrm{GeV}$. This restriction translates to an upper limit on the variable $E_{j_{1}}+E_{j_{2}}$ as a function of $m_{\chi}$ :

$$
E_{j_{1}}+E_{j_{2}} \leq \frac{s+m_{Z}^{2}-4 m_{\chi}^{2}}{2 \sqrt{s}}
$$

Since we are primarily interested in $\bar{\chi} \chi$ production associated with on-shell $Z \rightarrow j j / l l$, we shall use the condition on the total visible energy (19) as the selection cut for the rest of our analysis. The signatures where a pair of leptons appear in the final state due to the alternative decay modes of $Z$ boson, the differential cross-section distributions w.r.t. the observables $\Delta \phi_{l^{+} l^{-}}$and $E_{l^{+}}+E_{l^{-}}$, are similar to the observables $\Delta \phi_{j j}$ and $E_{j_{1}}+E_{j_{2}}$, which are obtained from the respective $E_{T}+2$ jets. Therefore, we adopt similar selection cut criteria on $E_{T}$ and $E_{l^{+}}+E_{l^{-}}$.
Based on the study of 1-D differential cross-section distributions at the given luminosity we select the two most sensitive kinematic observables say $E_{T}$ and either $\Delta \phi_{j j}$ or $\Delta \phi_{l^{+} l^{-}}$for the further analysis of two-dimensional differential cross-section distributions. We analyse the efficiency of the selection cut (19) through the double distributions of the simulated $Z$ associated DM pair-production events (for $m_{\chi}$ values at 75,225 and $325 \mathrm{GeV}$ ), which are then compared with the double differential cross-section distributions of the $\mathrm{SM}$ at the given integrated luminosity.

In Fig. 6, we exhibit the normalised two-dimensional differential cross section with the selection cuts in the $E_{T}$ and $\Delta \phi_{j j}$ plane for the background and the DM with $m_{\chi}=325$ $\mathrm{GeV}$. These 3D lego plots are generated from MadAnalysis 5 [59] at $\sqrt{s}=1 \mathrm{TeV}, \Lambda=1 \mathrm{TeV}$ and $\alpha_{S S}^{q}=1$. The bin width is $15 \mathrm{GeV}$ for the $Z_{T}$, while it is 0.1 for the $\Delta \phi_{i i}$ distribution.

We observe that the suppression of background processes due to the implementation of selection cuts enhances the sensitivity of the signal. We study the signal efficiency $(S / \sqrt{S+B})$ at $\sqrt{s}=1 \mathrm{TeV}$ with the integrated luminosity $1 \mathrm{ab}^{-1}$, and in Table 3 we compare the $3 \sigma$ reach of the cut-off $\Lambda$ at a given mass $m_{\chi}$ for the dominant signature $e^{+} e^{-} \rightarrow \mathbb{E}_{T}+2$ jets corresponding to the three representative masses of DM. 


\section{$5.2 \chi^{2}$ analysis}

In this subsection we study the sensitivity of the cut-off scale $\Lambda$ with the DM mass keeping the couplings of the DM bilinears with the SM fermionic pair to be unity. The $\chi^{2}$ analysis is performed with the sum of the variance of the differential bin events at a given integrated luminosity due to the presence of the new physics (NP) contribution over the SM events.

We define the $\chi^{2}$ for double distribution as

$$
\chi^{2}=\sum_{j=1}^{n_{1}} \sum_{i=1}^{n_{2}}\left(\frac{N_{i j}^{\mathrm{NP}}}{\sqrt{N_{i j}^{\mathrm{SM}+\mathrm{NP}}+\delta_{s y s}^{2}\left(N_{i j}^{\mathrm{SM}+\mathrm{NP}}\right)^{2}}}\right)^{2}
$$

where $N_{i j}^{\mathrm{NP}}$ are number of differential events given by New Physics and $N_{i j}^{\mathrm{SM}+\mathrm{NP}}$ is total number of differential events for the $(i j)$ th grid in double distribution. $\delta_{s y s}$ is the total systematic error in the measurement. Although the systematic uncertainty inclusive of the luminosity uncertainty is considered to be of the order of $0.3 \%$ in the literature [58], we have considered a conservative total systematic error to be of order of $1 \%$. Further, we find that the sensitivity of the cut-off $\Lambda$ can be increased with the increase in the luminosity as the cut-off scales $\sim \mathcal{L}^{1 / 8}$ for a given $\chi^{2}$. Thus, for $\sqrt{s}=1 \mathrm{TeV}$ and Luminosity $\mathcal{L}=1 \mathrm{ab}^{-1}$ the sensitivity of the signal will improve by $\approx 10 \%$.

From the $\chi^{2}$ analysis, we obtained the $3 \sigma$ contours in the $m_{\chi}-\Lambda$ plane corresponding to all the three processes in Figs. 7 and 8 . We present the estimation of the $3 \sigma$ sensitivity reach of the maximum value of the cut-off $\Lambda_{\max }$. that can be probed in ILC at $\sqrt{s}=500 \mathrm{GeV}$ and $1 \mathrm{TeV}$ with an integrated luminosity $\mathcal{L}=500 \mathrm{fb}^{-1}$ and $1 \mathrm{ab}^{-1}$, respectively, in Table 3 .

It is important to note that constraints on the cut-off $\Lambda$ for an appreciable range of fermion DM mass in Figs. 7 and 8 are lower than that of the centre-of-mass energy of the collider, which raises a pertinent question of the validity of the effective theory and our analysis in this mass region. However, the validation of these results in the domain of the effective field theory can be understood by re-writing the cut-off in terms of $\Lambda_{\text {Eff. }}$. Using Eqs. (2) and (4) we find that

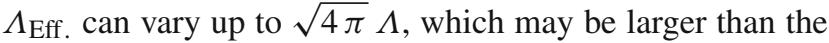
respective centre-of-mass energy of the collider.

We compare our results for the unpolarised beams at $\sqrt{s}=$ $500 \mathrm{GeV}$ and $\mathcal{L}=500 \mathrm{fb}^{-1}$ which are displayed in Fig. 7 with Fig. 4d and e of Ref. [53] corresponding to pseudo-scalar and axial-vector couplings respectively. We find that our analysis can provide a better kinematic reach $\left(m_{\chi}=20 \mathrm{GeV}\right)$ for the pseudo-scalar coupling. We can probe $\Lambda_{\max }$ up to $0.98 \mathrm{TeV}$, which is further improved to $1.07 \mathrm{TeV}$ with the enhanced integrated luminosity of $\mathcal{L}=1 \mathrm{ab}^{-1}$. However, for the case of axial-vector couplings we can only probe $\Lambda_{\max }$. up to 0.88 $\mathrm{TeV}$ as compared to $0.92 \mathrm{TeV}$ given in Fig. $4 \mathrm{e}$ of [53].
On the same note, we would like to compare our results for $\sqrt{s}=1 \mathrm{TeV}$ and $\mathcal{L}=1 \mathrm{ab}^{-1}$, which are displayed in Fig. 8 with Fig. $4 d$ and e of Ref. [53] corresponding to the pseudo-scalar and axial vector, respectively. We find that the sensitivity for the pseudo-scalar can be improved $\Lambda_{\max }$. 2.62 TeV in comparison to $2.3 \mathrm{TeV}$ given in Fig. 4d of [53]. Similarly, for axial-vector couplings, we agree with $\Lambda_{\max }$. given in Fig. 4e of [53].

\subsection{Effect of polarised beam}

We further study the sensitivity of $\Lambda$ dependence on $m_{\chi}$ with the polarised initial beams. The rate of pair production of the fermionic DM through scalar (SS) and pseudoscalar (PP) interactions can be enhanced by increasing the flux of right (left) handed electrons and right (left) handed positrons. Similarly VV and AA interaction of the fermionic DM can be enhanced by choosing the right (left) handed electron beam and left(right) handed positron beam. The background contribution from $t$-channel $W$ exchange diagram can be suppressed significantly by choosing right handed polarised electron beam which then leaves us with the following choices of polarisation combination w.r.t. helicity conserving (VV, AA) and helicity flipping (SS, PP) interactions, respectively:

1. $+80 \% e-$ and $-30 \% e+$;

2. $+80 \% e-$ and $+30 \% e+$.

We exhibit the $99 \%$ C.L. contours corresponding to the polarised initial $e^{-}$and $e^{+}$beams in Figs. 7 and 8 in the $m_{\chi}-\Lambda$ plane for all possible visible signatures associated with DM pair production at $\sqrt{s}=500 \mathrm{GeV}$ and $1 \mathrm{TeV}$ with an integrated luminosity of 250 and $500 \mathrm{fb}^{-1}$ respectively. We find that, for scalar and pseudo-scalar (vector and axialvector) DM couplings, we can probe $\Lambda_{\max }$. values up to 1.1 $\mathrm{TeV}(0.89 \mathrm{TeV})$ with polarised initial beams at an integrated Luminosity of $250 \mathrm{fb}^{-1}$. Similarly, we observe that the analysis with $\sqrt{s}=1 \mathrm{TeV}$ and an integrated luminosity of $1 \mathrm{ab}^{-1}$ the sensitivity of $\Lambda_{\max }$. can be improved to $3.13 \mathrm{TeV}$ (2.05 $\mathrm{TeV}$ ) for scalar and pseudo-scalar (vector and axial-vector) DM couplings.

We perform the combined $\chi^{2}$ analyses from all the three processes involving the DM pair production for both unpolarised and polarised initial beams. We compute the $99 \%$ confidence limit contours and plot them in Figs. 9 and 10 for $\sqrt{s}=500 \mathrm{GeV}$ and $1 \mathrm{TeV}$, respectively.

\subsection{Comparison with the existing analysis}

We present a comparison of our results with those that exist in the recent literature. 
Process 1: $e^{-} e^{+} \rightarrow 2$ jets $+E_{T}$
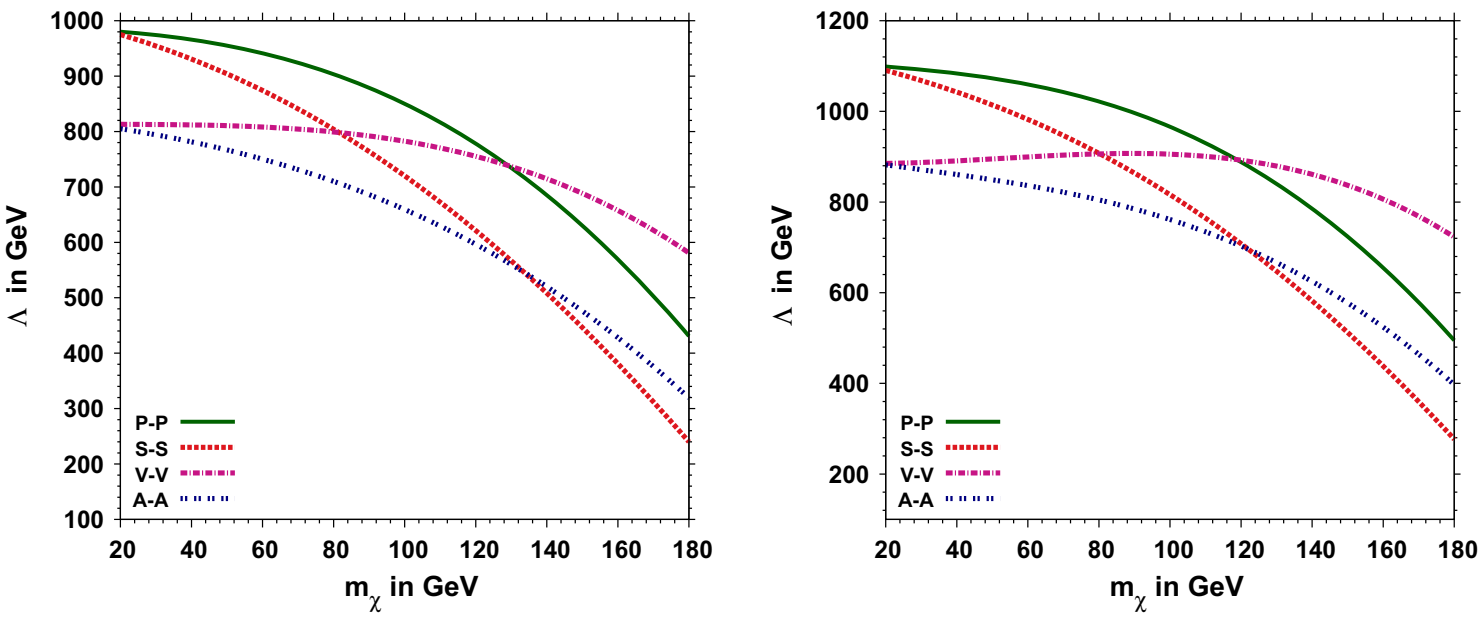

Process 2: $e^{+} e^{-} \rightarrow \mu^{+} \mu^{-}+E_{T}$
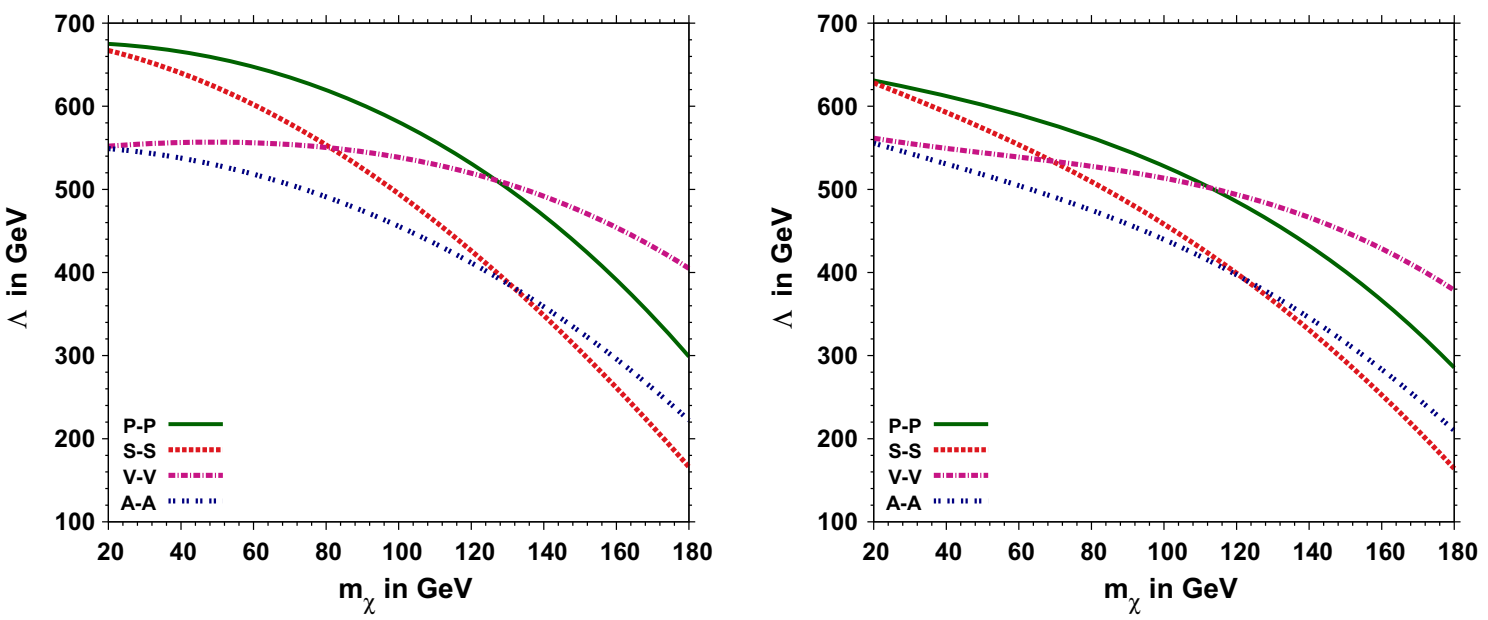

Process 3: $e^{+} e^{-} \rightarrow e^{+} e^{-}+\not E_{T}$

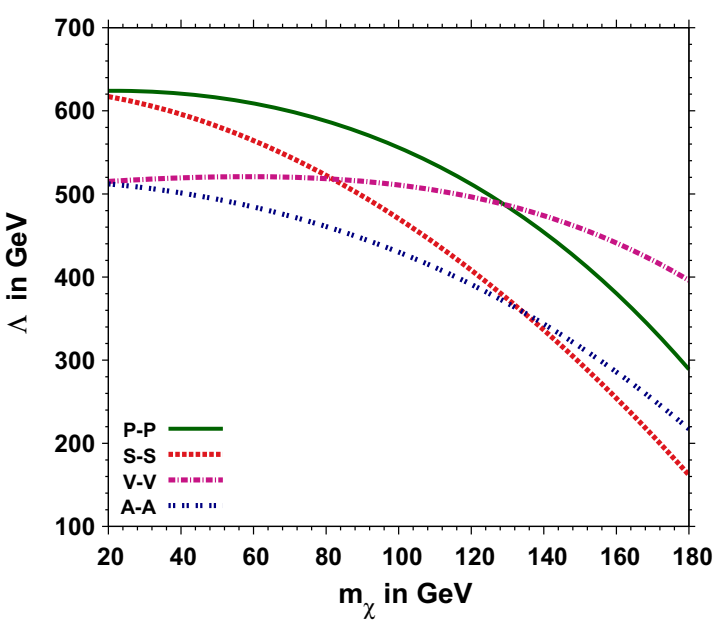

Fig. $799 \%$ confidence level contours in the $m_{\chi}$ and $\Lambda$ plane from the $\chi^{2}$ analyses of the respective final visible states at $\sqrt{s}=500 \mathrm{GeV}$ for the collider parameter choice given in the first and the second column of Table 1. The contours at the left correspond to the unpolarised beam

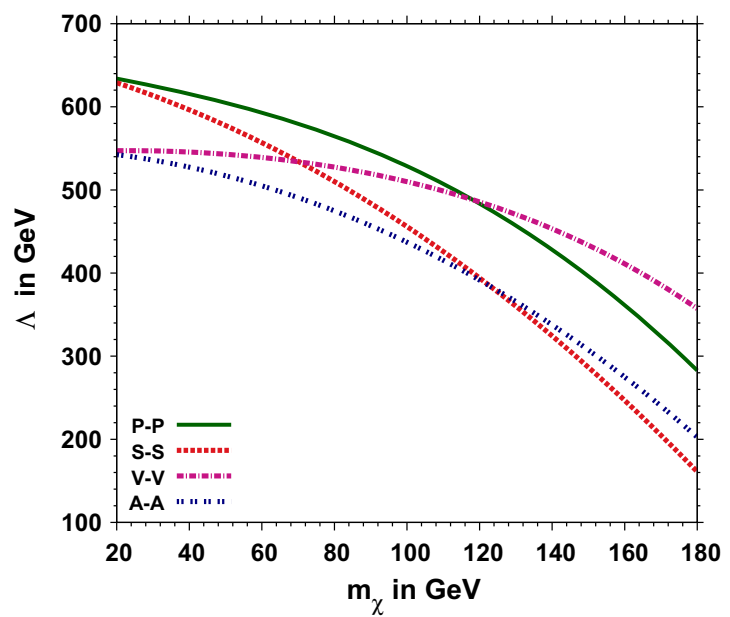

of $e^{-}$and $e^{+}$with an integrated luminosity of $500 \mathrm{fb}^{-1}$. The contours at the right correspond to 80 and $30 \%$ polarised beam for $e^{-}$and $e^{+}$, respectively, with an integrated luminosity of $250 \mathrm{fb}^{-1}$. The respective coupling of DM with the charged lepton $\alpha_{M N}^{l}$ is taken to be unity 
Process $1: e^{-} e^{+} \rightarrow 2$ jets $+E_{T}$
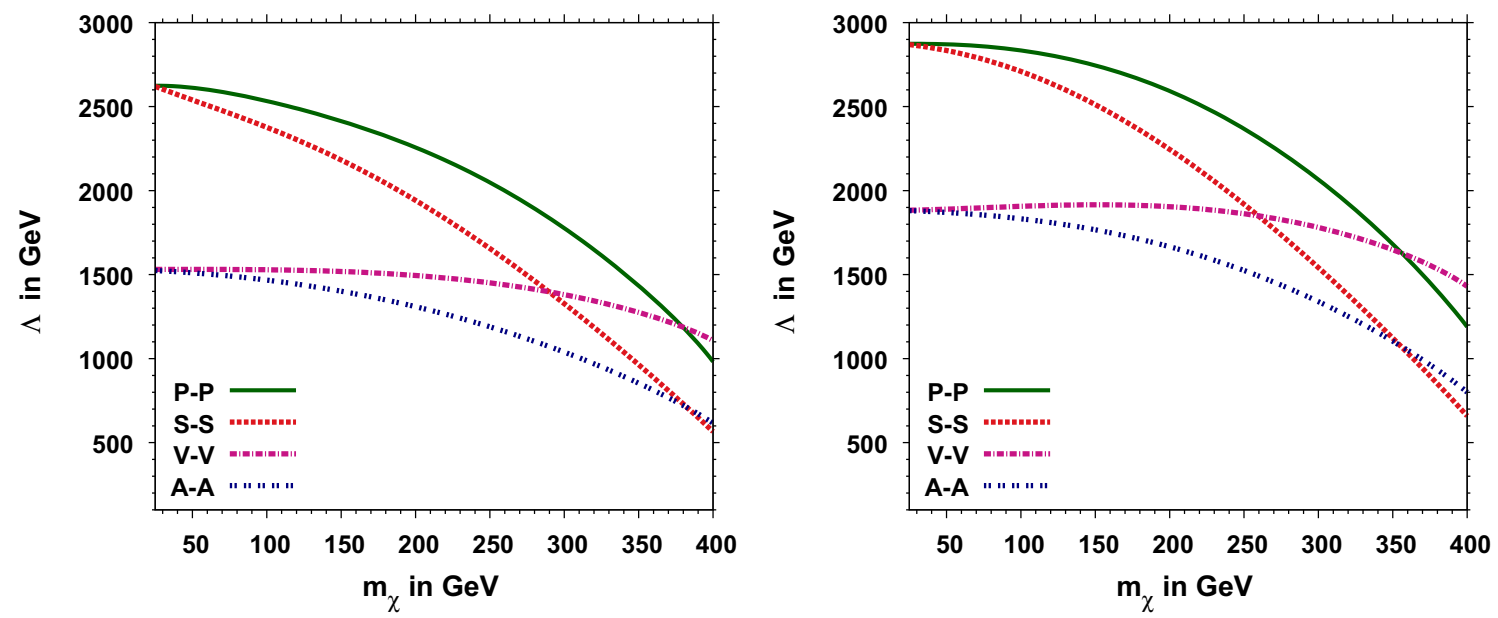

Process 2: $e^{+} e^{-} \rightarrow \mu^{+} \mu^{-}+\mathscr{E}_{T}$
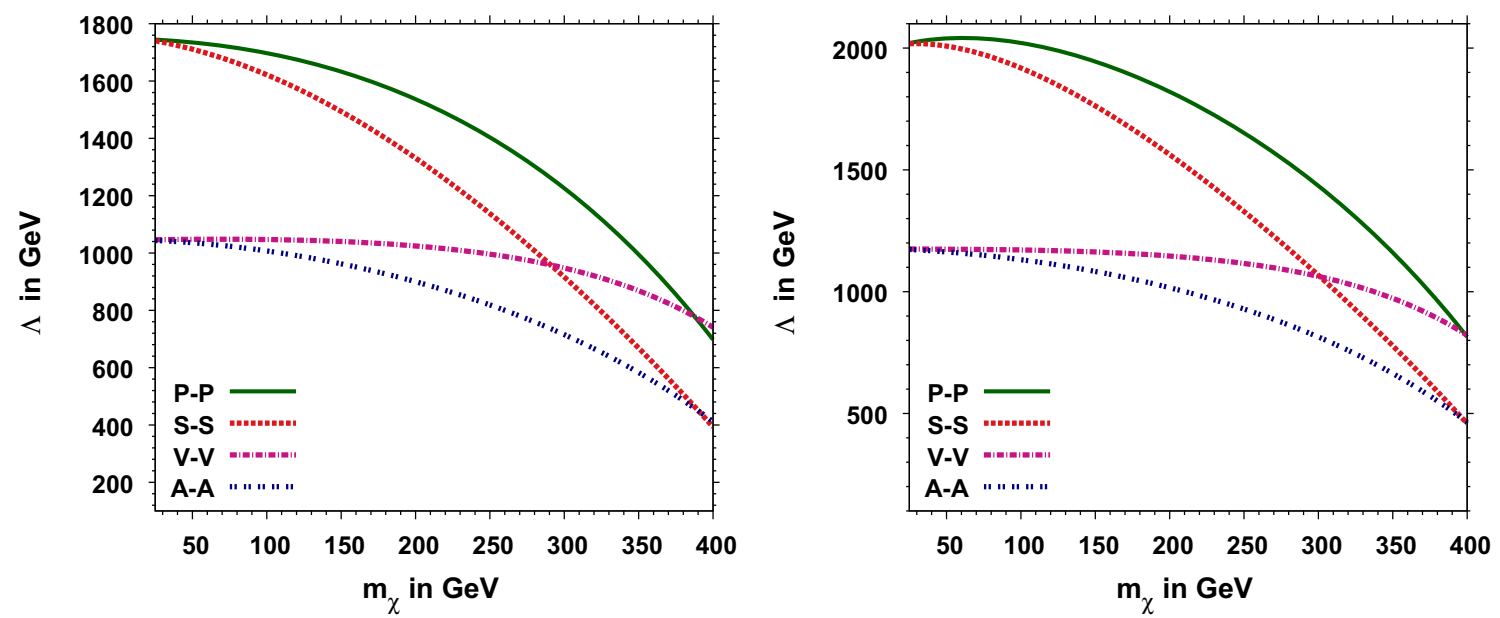

Process $3: e^{+} e^{-} \rightarrow e^{+} e^{-}+E_{T}$

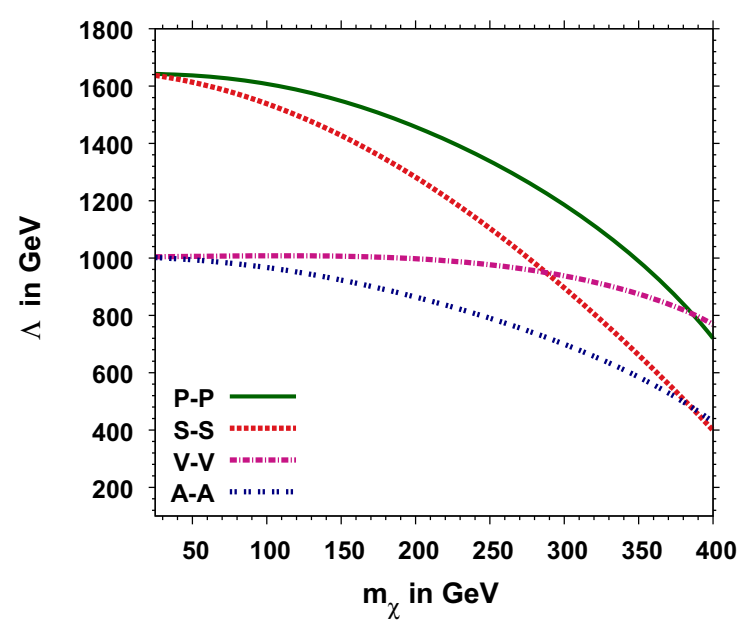

Fig. $899 \%$ confidence level contours in the $m_{\chi}$ and $\Lambda$ plane from the $\chi^{2}$ analyses of the respective final visible states at $\sqrt{s}=1 \mathrm{TeV}$ for the collider parameter choice given in the third and the fourth column of Table 1 . The contours at the left correspond to the unpolarised beam

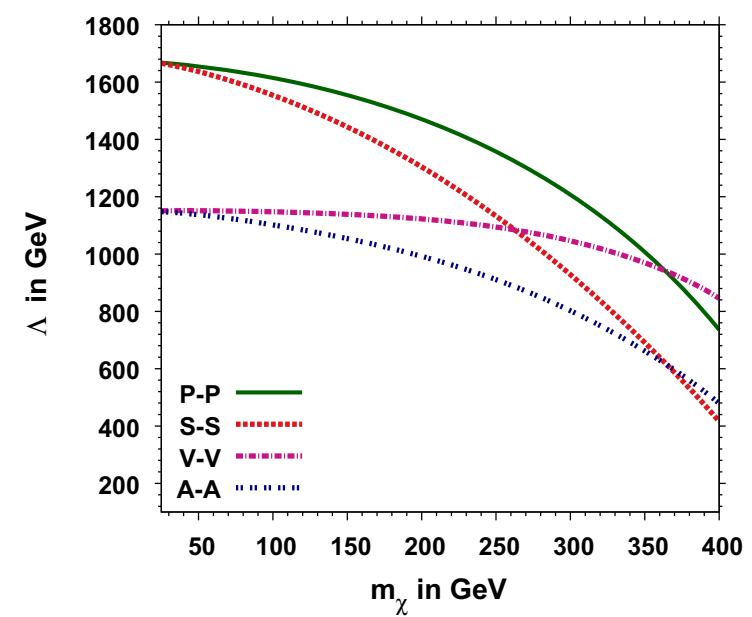

of $e^{-}$and $e^{+}$with an integrated luminosity of $1 \mathrm{ab}^{-1}$. The contours at the right correspond to 80 and $30 \%$ polarised beam for $e^{-}$and $e^{+}$, respectively, with an integrated luminosity of $500 \mathrm{fb}^{-1}$. The respective coupling of DM with the charged lepton $\alpha_{M N}^{l}$ is taken to be unity 


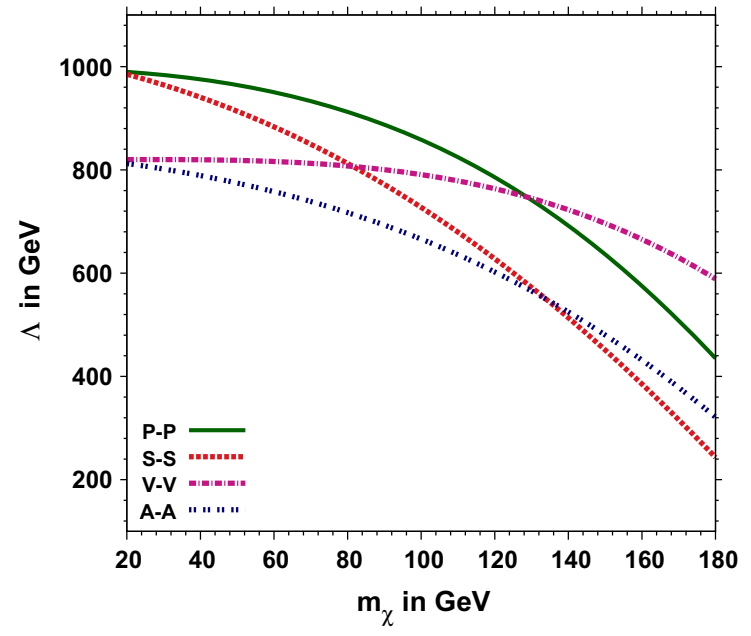

Fig. $999 \%$ confidence level contours in the $m_{\chi}$ and $\Lambda$ plane from the combined $\chi^{2}$ analyses of all the three visible modes at $\sqrt{s}=500 \mathrm{GeV}$ for the collider parameter choice given in the first and second column of Table 1 . The contours at the left correspond to the unpolarised beam

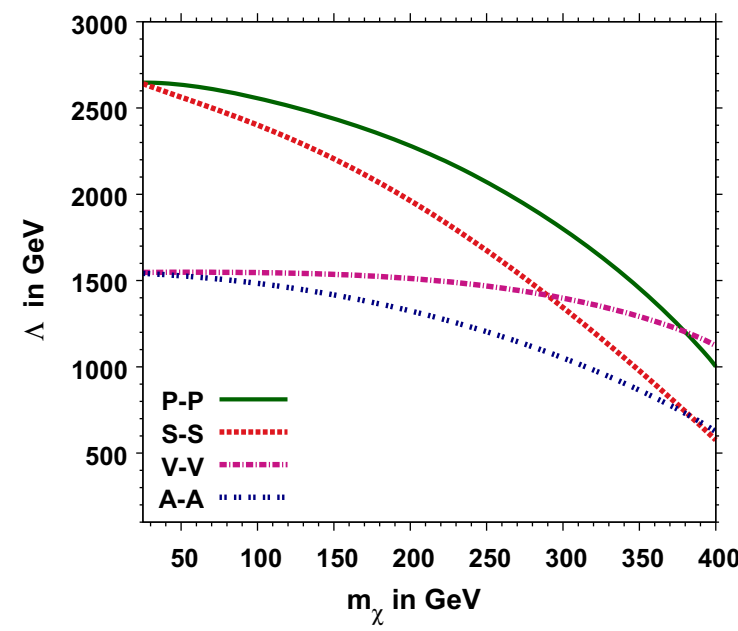

Fig. $1099 \%$ confidence level contours in the $m_{\chi}$ and $\Lambda$ plane from the combined $\chi^{2}$ analyses of all the three visible modes at $\sqrt{s}=1 \mathrm{TeV}$ for the collider parameter choice given in the third and fourth column of Table 1 . The contours at the left correspond to the unpolarised beam

\subsubsection{Sensitivity from mono-photon channel}

First, we make an overall comparison with the other complementary dominant DM production mono-photon channel studied in the Ref. [38]. We have calculated and verified the cross sections and significance of various interactions in the mono-photon channel with the cuts mentioned in [38].

We summarise and compare the sensitivity of the kinematic reach of the ILC on the cut-off $\Lambda_{\max }$ w.r.t. scalar, vector and axial-vector interactions of DM with the visible leptons in Table 4 corresponding to the mono- $Z$ channel and mono-photon signatures for both unpolarised and polarised

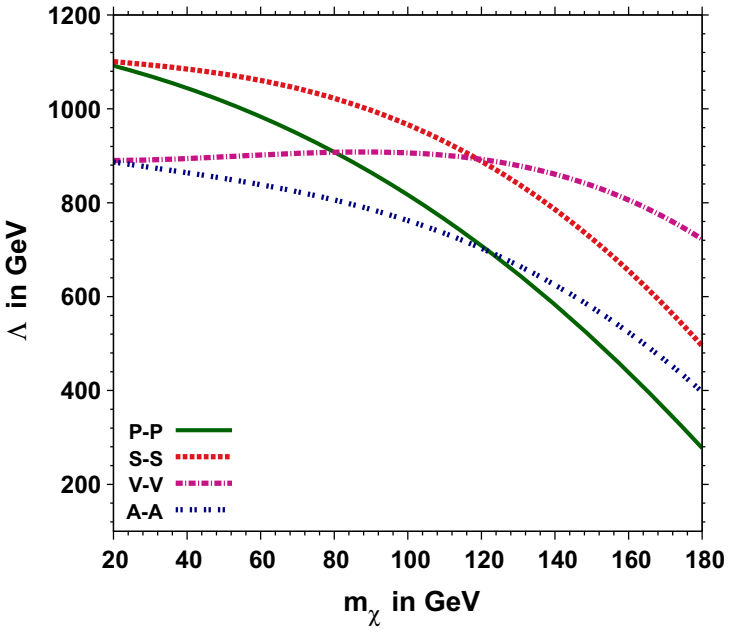

of $e^{-}$and $e^{+}$with an integrated luminosity of $500 \mathrm{fb}^{-1}$. The contours at the right correspond to 80 and $30 \%$ polarised beam for $e^{-}$and $e^{+}$ respectively with an integrated luminosity of $250 \mathrm{fb}^{-1}$. The respective coupling of DM with the charged lepton $\alpha_{M N}^{l}$ is taken to be unity

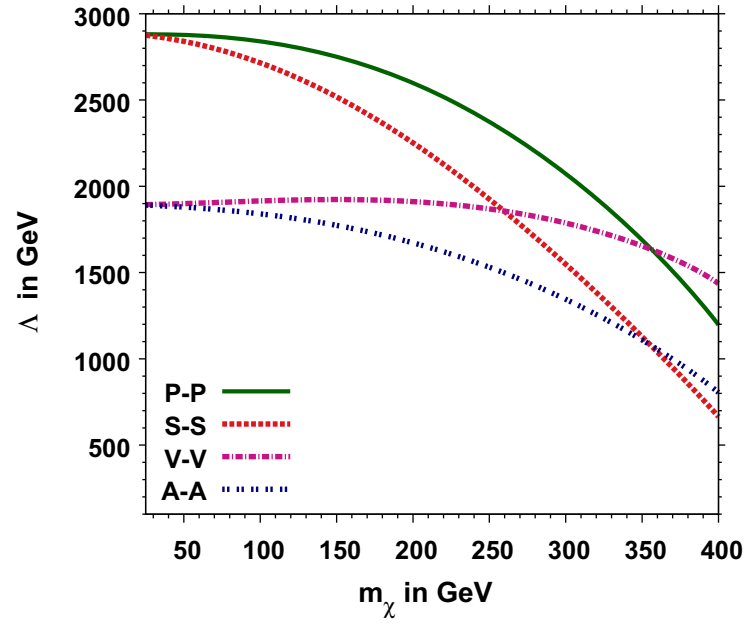

of $e^{-}$and $e^{+}$with an integrated luminosity of $1 \mathrm{ab}^{-1}$. The contours at the right correspond to 80 and $30 \%$ polarised beam for $e^{-}$and $e^{+}$, respectively, with an integrated luminosity of $500 \mathrm{fb}^{-1}$. The respective coupling of DM with the charged lepton $\alpha_{M N}^{l}$ is taken to be unity

initial beams. Our analysis shows that the sensitivity of $\Lambda$ is higher than that of the mono-photon channel, when mediated by the scalar coupling of the DM, specially in the lower $m_{\chi}$ region and behave as competitive DM production channel up to a DM mass of $\approx 275 \mathrm{GeV}$ for unpolarised initial beams. The restriction on the emerging angle of photon $\left|\cos \theta_{\gamma}\right| \leq 0.995$ opted in Ref. [38] reduced the $\gamma+E / T$ SM background appreciably and thus enhanced the signal efficiency.

It is important to mention that the enhancement in the sensitivity for the low $m_{\chi}$ region are obtained despite our conservative input for the systematic uncertainty $\approx 1 \%$ in contrast to that of $\approx 0.3 \%$ in Ref. [38]. 
Table 4 Comparison of the ILC kinematic reach on the $\Lambda_{\max }$ in $\mathrm{TeV}$ for four representative values of $m_{\chi}$ from the mono- $Z$ channel and mono- $\gamma$ channels corresponding to the scalar and vector interactions with the unpolarised and polarised initial beams at $\sqrt{s}=1 \mathrm{TeV}$. In columns II and III we give the $\Lambda_{\max }$ for the hadronic channel based on our two- dimensional $\chi^{2}$ analysis with $\delta_{\text {sys }}=1 \%$ and in columns IV and $\mathrm{V}$ we give the corresponding values of $\Lambda_{\max }$ for the mono-photon channel taken from [38]. The respective coupling of DM with the charged lepton $\alpha_{M N}^{e}$ is taken to be unity

\begin{tabular}{|c|c|c|c|c|c|c|c|c|}
\hline \multirow[b]{2}{*}{$\begin{array}{l}\text { Couplings } \\
\sqrt{s} \text { in } \mathrm{TeV} \\
\mathcal{L} \text { in } \mathrm{ab}^{-1} \\
\left(P_{e^{-}}, P_{e^{+}}\right) \\
m_{\chi} \text { in } \mathrm{GeV} \\
\end{array}$} & \multicolumn{2}{|c|}{$\begin{array}{l}\text { Mono- } Z \\
e^{+} e^{-} \rightarrow Z+E_{T} \\
\text { Unpolarised }\end{array}$} & \multicolumn{2}{|l|}{ Polarised } & \multicolumn{4}{|c|}{$\begin{array}{l}\text { Mono- } \gamma \\
e^{+} e^{-} \rightarrow \gamma+E_{T} \text { (Reference [38]) }\end{array}$} \\
\hline & $\begin{array}{l}\text { S-S } \\
1.0 \\
1 \\
(0,0)\end{array}$ & $\begin{array}{l}\mathrm{V}-\mathrm{V} \\
1.0 \\
1 \\
(0,0)\end{array}$ & $\begin{array}{l}S-S \\
1.0 \\
0.5 \\
(0.8,-0.5)\end{array}$ & $\begin{array}{l}\mathrm{V}-\mathrm{V} \\
1.0 \\
0.5 \\
(0.8,0.5)\end{array}$ & $\begin{array}{l}\text { S-S } \\
1.0 \\
1 \\
(0,0)\end{array}$ & $\begin{array}{l}\mathrm{V}-\mathrm{V} \\
1.0 \\
1 \\
(0,0)\end{array}$ & $\begin{array}{l}S-S \\
1.0 \\
0.5 \\
(0.8,-0.5)\end{array}$ & $\begin{array}{l}\mathrm{V}-\mathrm{V} \\
1.0 \\
0.5 \\
(0.8,0.5)\end{array}$ \\
\hline 25 & 2.62 & 1.60 & 2.88 & 1.9 & 2.0 & 2.14 & 3.66 & 4.0 \\
\hline 75 & 2.45 & 1.60 & 2.73 & 1.9 & 1.97 & 2.14 & 3.64 & 4.0 \\
\hline 225 & 1.91 & 1.58 & 2.10 & 1.94 & 1.78 & 2.10 & 3.25 & 3.98 \\
\hline 325 & 1.22 & 1.45 & 1.5 & 1.80 & 1.51 & 2.0 & 2.80 & 3.85 \\
\hline
\end{tabular}

Table 5 Efficiency $S / \sqrt{B}$ of the process $e^{+} e^{-} \rightarrow j j+E_{T}$ for $m_{\chi}$ $=10 \mathrm{GeV}$ and $\Lambda=1 \mathrm{TeV}$ at $\sqrt{s}=500 \mathrm{GeV}$ and an integrated luminosity $\mathcal{L}$ $=100 \mathrm{fb}^{-1}$, with unpolarised and polarised initial beams. The respective coupling of DM with the charged lepton $\alpha_{M N}^{e}$ is taken to be unity

\begin{tabular}{|c|c|c|c|c|}
\hline \multirow[b]{2}{*}{$\begin{array}{l}\text { Interactions } \\
\left(P_{e^{-}}, P_{e^{+}}\right)\end{array}$} & \multicolumn{2}{|c|}{ Unpolarised } & \multicolumn{2}{|l|}{ Polarised } \\
\hline & $\begin{array}{l}\mathrm{V}-\mathrm{V} \\
(0,0)\end{array}$ & $\begin{array}{l}\text { A-A } \\
(0,0)\end{array}$ & $\begin{array}{l}\mathrm{V}-\mathrm{V} \\
(0.8,-0.3)\end{array}$ & $\begin{array}{l}\mathrm{A}-\mathrm{A} \\
(0.8,-0.3)\end{array}$ \\
\hline \multicolumn{5}{|c|}{ Reference [52] } \\
\hline \multicolumn{5}{|l|}{$m_{\chi}$ in $\mathrm{GeV}$} \\
\hline 10 & 0.96 & 0.95 & 1.77 & 1.76 \\
\hline 140 & 0.55 & 0.5 & 0.84 & 0.84 \\
\hline \multicolumn{5}{|l|}{ Our analysis } \\
\hline \multicolumn{5}{|l|}{$m_{\chi}$ in $\mathrm{GeV}$} \\
\hline 10 & 0.99 & 0.98 & 1.67 & 1.66 \\
\hline 140 & 0.76 & 0.72 & 1.6 & 1.57 \\
\hline
\end{tabular}

\subsubsection{Results from mono- $Z$ channels}

Next, we compare our results with the existing analysis on the DM production channels in association with $Z$ boson which decays to pair of light quarks, electrons and muons.

DM interactions mediated through the vector and axialvector couplings are compared with the results of Ref. [52]. Unlike our analysis, they have ignored the background contribution from the $t$-channel $W^{+} / W^{-}$diagrams as well as the photon exchange diagrams. Regardless of the higher standard model background our cuts profile exhibit a marginal improvement in $S / \sqrt{B}$ for the unpolarised case as shown in Table 5. We found that the cut $10^{\circ}<\theta_{Z}<170^{\circ}$ opted by the authors of Ref. [52] is quite effective for the low $m_{\chi}$ region as it filters the background consisting of the highly boosted neutrino-antineutrino pair in the forward direction, without disturbing the signal and as a consequence their anal- ysis gives the better signal efficiency for the polarised beams. We observe that our cut strategy is preferable for $50 \mathrm{GeV}$ $\leq m_{\chi} \leq 200 \mathrm{GeV}$ region and accordingly the signal efficiency improve with the increasing DM mass.

On basis of $\chi^{2}$ analysis we marginally improve the upper limit $\Lambda_{\max }$ ( at $m_{\chi}=10 \mathrm{GeV}$ ) corresponding to the unpolarised initial beams. However, it should be noted that the authors of Ref. [52] have assumed

(a) $\alpha_{\mathrm{em}}^{-1}$ to be 137 at $\sqrt{s}=500 \mathrm{GeV}$ or $1 \mathrm{TeV}$ instead of 127.9 , thus leading to the gross under-estimation of the background events. Rectifying the error will further bring down the efficiency of the sensitivity of their analysis.

(b) zero percent systematic uncertainty. Our analysis has been performed with the conservative $1 \%$ systematic uncertainty.

We also compare the significance of the mono- $Z$ channel processes in the hadronic decay mode with those given in Ref. [53] at $\sqrt{s}=500 \mathrm{GeV}$ for $\alpha_{M N}^{e}=1$. We summarise this in Table 6 for some representative values of $m_{\chi}$ and $\Lambda$. We find that the our cut strategy and analysis shows enhancement in the signal significance w.r.t. the pseudo-scalar and axial-vector interactions of DM with charged leptons for both unpolarised and polarised initial beams.

We observe that the representative values of $\Lambda$ chosen for comparison are again appreciably lower than that of the centre-of-mass energy of the collider. For clarity and better understanding, we would like to emphasise that this is allowed and that the applicability of the EFT within the perturbative limit is valid as long as $\sqrt{s}<\Lambda_{\text {Eff. }}$. Following the same argument for the coupling $\alpha_{M N}^{f} \sim 1$ as given in Eq. (4), the representative choices of $\Lambda=400$ and $280 \mathrm{GeV}$ can be read as $\Lambda_{\text {Eff. }} \lesssim 1.4 \mathrm{TeV}$ and $0.92 \mathrm{TeV}$ corresponding to 
Table 6 Efficiency $S / \sqrt{S+B}$ of the process $e^{+} e^{-} \rightarrow j j+\mathbb{E}_{T}$ at $\sqrt{s}$ $=500 \mathrm{GeV}$ and an integrated luminosity $\mathcal{L}=100 \mathrm{fb}^{-1}$ corresponding to the benchmark points mentioned in Ref. [53] for both unpolarised and polarised initial beams. The respective coupling of DM with the charged lepton $\alpha_{M N}^{e}$ is taken to be unity

\begin{tabular}{llllll}
\hline & \multicolumn{2}{l}{ Unpolarised } & & Polarised & \\
\cline { 2 - 3 } \cline { 6 - 6 } Interactions & $\mathrm{P}-\mathrm{P}$ & $\mathrm{A}-\mathrm{A}$ & & $\mathrm{P}-\mathrm{P}$ & $\mathrm{A}-\mathrm{A}$ \\
$m_{\chi}$ in $\mathrm{GeV}$ & 120 & 150 & & 120 & 150 \\
$\Lambda$ in $\mathrm{GeV}$ & 400 & 280 & & 400 & 280 \\
$\left(P_{e^{-}}, P_{e^{+}}\right)$ & $(0,0)$ & $(0,0)$ & & $(0.8,-0.3)$ & $(0.8,-0.3)$ \\
\hline Reference [53] & 18.7 & 12.3 & & 34.4 & 23.0 \\
Our analysis & 21.13 & 18.45 & & 37.14 & 28.74 \\
\hline
\end{tabular}

$\mathrm{P}-\mathrm{P}$ and $\mathrm{A}-\mathrm{A}$ interactions respectively which are higher than the centre-of-mass energy $\sqrt{s}=500 \mathrm{GeV}$.

\section{Summary and outlook}

The fact that a massive $\mathcal{O}(100 \mathrm{GeV})$ stable particle $\chi$ with near-weak scale interactions can constitute an attractive candidate for the dark matter in the universe (while satisfying all competing constraints from the relic density, large-scale structure formation as well as myriad other cosmological and astrophysical observations) is well known. While the correct relic abundance can be reproduced by ensuring the right annihilation cross sections into any of the SM particles, direct detection of the DM candidate largely hinges on its having unsuppressed interactions with the first-generation quarks.

It is then of interest to consider the possibility that $\chi$ has very suppressed (if any) interactions with quarks and gluons. Not only would the direct-detection experiments have very little sensitivity to such particles, their pair-production cross section at the LHC would be suppressed too. Under such circumstances, though, the DM must have significant couplings to at least some of the leptons and/or the electroweak gauge bosons.

In this article, we have investigated the electrophilic and/or leptophilic spin-1/2 DM using an effective field theory approach and examined in detail its discovery potential at ILC. We found that the signal efficiency has been improved upon the previous ILC studies on the mono- $Z+\mathbb{E}_{T}$ signals for $m_{\chi}$ varying between 20 and $400 \mathrm{GeV}$ by making an effective choice of cuts flow and using the correct value of SM input parameters. We have further enhanced the sensitivity of the kinematic reach of the model parameter $\Lambda$ for a given $m_{\chi}$ and unity coupling by performing the $\chi^{2}$ test using the two-dimensional distributions of the kinematic observables obtained from the simulated SM background and new physics DM production processes followed by parton showering and hadronisation.
We obtained the upper bound on the cut-off $\Lambda$ for the given range of electrophilic and/or leptophilic DM mass $m_{\chi}$ from the relic density constraints which is displayed in the Fig. 1. Using these upper bounds on $\Lambda$ for a given $m_{\chi}$, we estimated the thermally averaged annihilation indirect-detection cross section and leptophilic DM direct-detection scattering cross section and are displayed in Figs. 2 and 3, respectively. We find that the present experimental limits in the respective searches not only favour the allowed parameter space from the relic density but also constrain the DM model by providing the lower bound on the $\Lambda_{\min }$ for a given DM mass $m_{\chi}$.

If the couplings to the charged leptons would be unsuppressed, the linear collider could play an interesting role in establishing the existence of such a $\chi$. Using the allowed coupling to the electron, this has traditionally been undertaken using the mono-photon (accompanied by missing energymomentum) channel. We have here investigated the complementary channel, namely $e^{+} e^{-} \rightarrow \chi \bar{\chi}+f \bar{f}$, where $f$ is any of the light charged fermions (jets, electrons and muons) and exhibited the significance of these processes with basic kinematic cuts in Table 4.

We analysed the sensitivity of DM scalar and vector couplings through the one- and two-dimensional normalised differential kinematic distributions corresponding to the most dominant signature $e^{+} e^{-} \rightarrow j j+E_{T}$ in Figs. 4, 5 and 6, respectively. $99 \%$ C.L. contours for various sets of run parameters based on the $\chi^{2}$ analysis of these differential distributions are shown in Figs. 7, 8. Combining the $\chi^{2}$ analysis, from all the three processes corresponding to $\sqrt{s}=500 \mathrm{GeV}$ and $1 \mathrm{TeV}$ further improves the sensitivity of the cut-off for given DM mass and this is shown in Figs. 9 and 10, respectively. We find that a specific choice of the initial beam polarisation enhances the sensitivity of the cut-off for the scalar and vector couplings of DM.

On translating the kinematic reach of the collider on $\Lambda$ in terms of $\Lambda_{\text {Eff. at }} \sqrt{s}=500 \mathrm{GeV}$ and $1 \mathrm{TeV}$, respectively, our study show that the analysis of dimension-six operators in EFT can provide an early indication of the nature of leptophilic and/or electrophilic DM for the mass range $20 \mathrm{GeV}-$ $400 \mathrm{GeV}$ along with an idea of the scale associated with this effective theory. Our analysis also concludes that the cosmologically allowed $m_{\chi}-\Lambda_{\text {Eff. }}$ region corresponding to these operators can be probed with higher sensitivity through the important complementary channel $Z+Z_{T}$ along with the dominant mono-photon signal.

We hope this study will be useful in studying the physics potential of the ILC in context to dark matter searches.

Acknowledgements We thank Debajyoti Choudhury for useful discussions and comments on the manuscript. We also thank Hrishabh Bharadwaj for verifying some of our results. SD acknowledges the par- 
tial financial support from the CSIR Grant no. 03(1340)/15/EMR-II. BR and DS acknowledge the UGC for partial financial support. SD and BR would like to thank IUCAA, Pune, where part of this work was completed, for hospitality.

Open Access This article is distributed under the terms of the Creative Commons Attribution 4.0 International License (http://creativecomm ons.org/licenses/by/4.0/), which permits unrestricted use, distribution, and reproduction in any medium, provided you give appropriate credit to the original author(s) and the source, provide a link to the Creative Commons license, and indicate if changes were made.

Funded by SCOAP ${ }^{3}$.

\section{References}

1. M. Milgrom, A modification of the Newtonian dynamics as a possible alternative to the hidden mass hypothesis. Astrophys. J. 270, 365 (1983)

2. V.C. Rubin, W.K. Ford Jr., Rotation of the Andromeda Nebula from a spectroscopic survey of emission regions. Astrophys. J. 159, 379 (1970)

3. L.A. Moustakas, R.B. Metcalf, Detecting dark matter substructure spectroscopically in strong gravitational lenses. Mon. Not. R. Astron. Soc. 339, 607 (2003). arXiv:astro-ph/0206176

4. E. van Uitert et al, Constraints on the shapes of galaxy dark matter haloes from weak gravitational lensing. Astron. Astroph. 545 A71 (2012). arXiv:1206.4304

5. D. Clowe, M. Bradac, A.H. Gonzalez, M. Markevitch, S.W. Randall, C. Jones, D. Zaritsky, A direct empirical proof of the existence of dark matter. Astrophys. J. 648, L109 (2006). arXiv:astro-ph/0608407

6. G. Hinshaw et al., [WMAP Collaboration], "Nine-year Wilkinson microwave anisotropy probe (WMAP) observations: cosmological parameter results". Astrophys. J. Suppl. 208, 19 (2013). arXiv:1212.5226 [astro-ph.CO]

7. M.G. Abadi, J.F. Navarro, M. Steinmetz, V.R. Eke, Simulations of galaxy formation in a lambda CDM universe. 2. The fine structure of simulated galactic disks. Astrophys. J. 597, 21 (2003). arXiv:astro-ph/0212282

8. P.A.R. Ade et al. [Planck Collaboration], Planck 2015 results. XIII. Cosmological parameters. arXiv:1502.01589 [astro-ph.CO]

9. D.B. Thomas, M. Kopp, C. Skordis, Constraining dark matter properties with cosmic microwave background observations. arXiv:1601.05097 [astro-ph.CO]

10. J.M. Overduin, P.S. Wesson, Phys. Rept. 402, 267 (2004). doi:10. 1016/j.physrep.2004.07.006. arXiv:astro-ph/0407207

11. J.L. Feng, Ann. Rev. Astron. Astrophys. 48, 495 (2010). doi:10. 1146/annurev-astro-082708-101659. arXiv:1003.0904 [astroph.CO]

12. R. Bernabei et al., Final model independent result of DAMA/LIBRA-phase1. Eur. Phys. J. C 73, 2648 (2013). arXiv:1308.5109 [astro-ph.GA]

13. C.E. Aalseth et al., CoGeNT collaboration. Phys. Rev. D 88, 012002 (2013). doi:10.1103/PhysRevD.88.012002. arXiv:1208.5737 [astro-ph.CO]

14. G. Angloher et al. [CRESST collaboration]. arXiv:1612.07662 [hep-ex]

15. E. Aprile et al., XENON collaboration. Phys. Rev. D 94(9), 092001 (2016). doi:10.1103/PhysRevD.94.092001. arXiv:1605.06262 [astro-ph.CO]

16. Y. Yang, PandaX-II collaboration. arXiv:1612.01223 [hep-ex]

17. A. Tan et al., PandaX-II collaboration. Phys. Rev. Lett. 117(12), 121303 (2016). doi:10.1103/PhysRevLett.117.121303. arXiv:1607.07400 [hep-ex]
18. D.S. Akerib et al. arXiv: 1608.07648 [astro-ph.CO]

19. M. Ackermann et al., Fermi-LAT collaboration. Phys. Rev. Lett. 115(23), 231301 (2015). doi:10.1103/PhysRevLett.115.231301. arXiv:1503.02641 [astro-ph.HE]

20. A. Albert et al., Fermi-LAT and DES collaborations. Astrophys. J. 834(2), 110 (2017). doi:10.3847/1538-4357/834/2/110. arXiv: 1611.03184 [astro-ph.HE]

21. O. Adriani et al., PAMELA collaboration. Nature 458, 607 (2009). doi:10.1038/nature07942. arXiv:0810.4995 [astro-ph]

22. M. Aguilar et al., AMS collaboration. Phys. Rev. Lett. 110, 141102 (2013). doi:10.1103/PhysRevLett.110.141102

23. B. Bhattacherjee, D. Choudhury, K. Harigaya, S. Matsumoto, M.M. Nojiri, Model independent analysis of interactions between dark matter and various quarks. JHEP 1304, 031 (2013). arXiv:1212.5013 [hep-ph]

24. J. Goodman, M. Ibe, A. Rajaraman, W. Shepherd, T.M. Tait et al., Constraints on dark matter from colliders. Phys. Rev. D 82, 116010 (2010). arXiv:1008.1783

25. L .M. Carpenter, A. Nelson, C. Shimmin, T .M. Tait, D. Whiteson, Collider searches for dark matter in events with a Z boson and missing energy. Phys. Rev. D87(7), 074005 (2013). arXiv:1212.3352 [hep-ph]

26. A.A. Petrov, W. Shepherd, Searching for dark matter at LHC with Mono-Higgs production. Phys. Lett. B 730, 178-183 (2014). arXiv:1311.1511 [hep-ph]

27. L. Carpenter, A. DiFranzo, M. Mulhearn, C. Shimmin, S. Tulin et al., Mono-Higgs-boson: a new collider probe of dark matter. Phys. Rev. D89(7), 075017 (2014). arXiv:1312.2592 [hep-ph]

28. A. Berlin, T. Lin, L.-T. Wang, Mono-Higgs detection of dark matter at the LHC. JHEP 1406, 078 (2014). arXiv:1402.7074 [hep-ph]

29. T. Lin, E .W. Kolb, L.-T. Wang, Probing dark matter couplings to top and bottom quarks at the LHC. Phys. Rev. D88(6), 063510 (2013). arXiv:1303.6638 [hep-ph]

30. Y. Bai, P.J. Fox, R. Harnik, The tevatron at the frontier of dark matter direct detection. JHEP 1012, 048 (2010). arXiv: 1005.3797

31. A. Birkedal, K. Matchev, M. Perelstein, Dark matter at colliders: a model independent approach. Phys. Rev. D 70, 077701 (2004). arXiv:hep-ph/0403004

32. Y. Gershtein, F. Petriello, S. Quackenbush, K.M. Zurek, Discovering hidden sectors with mono-photon $Z^{\prime}$ searches. Phys. Rev. D 78, 095002 (2008). arXiv:0809.2849 [hep-ph]

33. J.L. Feng, S. Su, F. Takayama, Phys. Rev. Lett. 96, 151802 (2006). doi:10.1103/PhysRevLett.96.151802. arXiv:hep-ph/0503117

34. P.J. Fox, R. Harnik, J. Kopp, Y. Tsai, LEP shines light on dark matter. Phys. Rev. D 84, 014028 (2011). arXiv:1103.0240 [hepph]

35. Y. Bai, T.M. Tait, Searches with mono-leptons. Phys. Lett. B 723, 384-387 (2013). arXiv:1208.4361 [hep-ph]

36. A. Crivellin, U. Haisch, A. Hibbs, LHC constraints on gauge boson couplings to dark matter. arXiv: 1501.00907

37. F.J. Petriello, S. Quackenbush, K.M. Zurek, The invisible $Z^{\prime}$ at the CERN LHC. Phys. Rev. D 77, 115020 (2008). arXiv:0803.4005 [hep-ph]

38. Y.J. Chae, M. Perelstein, Dark matter search at a linear collider: effective operator approach. JHEP 1305, 138 (2013). arXiv:1211.4008 [hep-ph]

39. H. Dreiner, M. Huck, M. KrÃd'mer, D. Schmeier, J. Tattersall, Phys. Rev. D 87(7), 075015 (2013). arXiv:1211.2254 [hep-ph]

40. Z.H. Yu, Q.S. Yan, P.F. Yin, Phys. Rev. D 88(7), 075015 (2013). doi:10.1103/PhysRevD.88.075015. arXiv:1307.5740 [hep-ph]

41. C. Bartels, J. List. eConf C 0705302, COS02 (2007). arXiv:0709.2629 [hep-ex]

42. K. Griest, D. Seckel, Three exceptions in the calculation of relic abundances. Phys. Rev. D 43, 3191 (1991) 
43. J.M. Zheng, Z.H. Yu, J.W. Shao, X.J. Bi, Z. Li, H.H. Zhang, Constraining the interaction strength between dark matter and visible matter: I. fermionic dark matter. Nucl. Phys. B 854, 350 (2012). arXiv:1012.2022 [hep-ph]

44. D.J. Fixsen, Astrophys. J. 707, 916 (2009). arXiv:0911.1955 [astroph.CO]

45. C.L. Bennett et al., WMAP collaboration. Astrophys. J. Suppl. 208, 20 (2013). arXiv:1212.5225 [astro-ph.CO]

46. N.D. Christensen, C. Duhr, Comput. Phys. Commun. 180, 1614 (2009). arXiv:0806.4194 [hep-ph]

47. G. Belanger, F. Boudjema, A. Pukhov, A. Semenov, Comput. Phys. Commun. 149, 103 (2002). doi:10.1016/S0010-4655(02)00596-9. arXiv:hep-ph/0112278

48. M. Backovic, K. Kong, M. McCaskey, Phys. Dark Univ. 5-6, 18 (2014). doi:10.1016/j.dark.2014.04.001. arXiv:1308.4955 [hep$\mathrm{ph}]$

49. M. Backovic, A. Martini, K. Kong, O. Mattelaer, G. Mohlabeng, AIP Conf. Proc. 1743, 060001 (2016). doi:10.1063/1.4953318. arXiv:1509.03683 [hep-ph]

50. J. Kopp, V. Niro, T. Schwetz, J. Zupan, DAMA/LIBRA and leptonically interacting dark matter. Phys. Rev. D 80, 083502 (2009). arXiv:0907.3159 [hep-ph]
51. E. Aprile et al., [XENON100 collaboration] Exclusion of leptophilic dark matter models using XENON100 electronic recoil data. Science 349(6250), 851 (2015)

52. W. Neng, S. Mao, L. Gang, M. Wen-Gan, Z. Ren-You, G. Jian-You, Eur. Phys. J. C 74(12), 3219 (2014). arXiv:1403.7921 [hep-ph]

53. Z.-H. Yu, X.-J. Bi, Q.-S. Yan, P.-F. Yin, Dark matter searches in the mono- $Z$ channel at high energy $e^{+} e^{-}$colliders. Phys. Rev D90(5), 055010 (2014). arXiv:1404.6990 [hep-ph]

54. J. Alwall et al., JHEP 1407, 079 (2014). doi:10.1007/ JHEP07(2014)079. arXiv:1405.0301 [hep-ph]

55. T. Sjostrand, S. Mrenna, P.Z. Skands, JHEP 0605, 026 (2006). arXiv: hep-ph/0603175

56. PGS-4, J. Conway et al. http://www.physics.ucdavis.edu/ $\sim$ conway/research/software/pgs/pgs4-general.htm

57. T. Behnke et al. arXiv:1306.6329 [physics.ins-det]

58. T. Behnke et al., The international linear collider technical design report—volume 1: executive summary. arXiv:1306.6327 [physics.acc-ph]

59. E. Conte, B. Fuks, G. Serret, Comput. Phys. Commun. 184, 222 (2013). doi:10.1016/j.cpc.2012.09.009. arXiv:1206.1599 [hep-ph] 